\title{
Itinéraires féminins de sortie de la violence conjugale
}

Routes out of domestic violence

Nadine Lefaucheur, Joëlle Kabile et Léoncine Ozier-Lafontaine

\section{(2) OpenEdition}

Édition électronique

URL : http://journals.openedition.org/plc/868

DOI : $10.4000 /$ plc. 868

ISSN : 2117-5209

Éditeur

L'Harmattan

Édition imprimée

Date de publication : 1 janvier 2012

Pagination : 199-238

ISBN : 978-2-296-55-856-4

ISSN : $1279-8657$

\section{Référence électronique}

Nadine Lefaucheur, Joëlle Kabile et Léoncine Ozier-Lafontaine, « Itinéraires féminins de sortie de la violence conjugale », Pouvoirs dans la Caraibe [En ligne], 17 | 2012, mis en ligne le 27 janvier 2012, consulté le 19 avril 2019. URL : http://journals.openedition.org/plc/868 ; DOI : 10.4000/plc.868 


\title{
ITINÉRAIRES FÉMININS DE SORTIE DE LA VIOLENCE CONJUGALE
}

\author{
Nadine LEFAUCHEUR \\ Sociologue \\ Membre associé du CRPLC \\ Université des Antilles et de la Guyane
}

\author{
Joëlle KABILE \\ Doctorante \\ Membre associé du CRPLC \\ Université des Antilles et de la Guyane
}

\section{Léoncine OZIER-LAFONTAINE}

Doctorante

Conservatoire national des arts et métiers

\section{I. - « TROP, C'EST TROP » : PRISE DE CONSCIENCE ET DÉCLIC}

Le processus de sortie de la violence commence généralement par ce que les femmes décrivent souvent comme un déclic, comme «la goutte d'eau qui fait déborder le vase », car «trop, c'est trop !».

Le déclic - que le Petit Robert définit comme «le déclenchement d'un processus psychologique »- marque une rupture nette dans la trajectoire d'acceptation de la violence. C'est l'instant où la femme comprend qu'elle ne supportera plus les violences qu'elle endure. Cela ne signifie pas qu'elle ne se considère plus comme une victime, mais, au contraire, qu'elle se regarde enfin véritablement comme telle et qu'elle peut donc envisager des stratégies de sortie de la situation de violence.

Le déclic peut se produire lors d'un événement qui ne diffère pas ou ne diffère que peu des violences ordinairement subies par la femme, mais qui est «la goutte de trop », sans doute parce qu'il est très chargé symboliquement et fait écho à des aspirations profondes dont le comportement du conjoint, incompréhensible et illégitime pour elle, sonne le deuil.

Nathalie, d'origine étrangère, qui vivait depuis une dizaine d'années avec un Martiniquais très violent dans la famille de celui-ci, avait réussi à trouver une petite maison indépendante, où elle escomptait que les problèmes du couple s'arrangeraient. Le Noël dont elle espérait tant est au contraire l'occasion d'une recrudescence de la violence qu'elle ne peut comprendre : 
J'ai jamais trouvé un chance pour parler parce que j'ai voulu laisser. J'ai marre, j'ai marre... En 2005, on a trouvé an dernier volée avec un sapin, c'était la Noël, j'ai acheté un petit sapin pour décorer la maison et li pa ka ba mwen lajen, mais comme j'ai trouvé un p'tit djob en noir - j'ai toujours astiqué la maison, toujours fait la maison belle, j'ai dit 'peut-être un jour je vais peut-être inviter sa famille, la famille de JM, peut-être ma famille peut venir, on sait pas, mais quand même pour la première fois, j'ai resté en Martinique, je veux faire ma maison belle', mais c'est le premier la Noël, j'ai trouvé an bel volée. Pourquoi, je sais même pas .

Nathalie est frappée à l'occasion d'une fête religieuse et familiale importante, qu'elle passe pour la première fois seule avec son conjoint et son enfant; elle est frappée avec un objet emblématique de Noël, le sapin, qu'elle a acheté elle-même, grâce à ce qu'elle a réussi à gagner doublement au noir : un djob non déclaré par l'employeur, mais également effectué en cachette de son compagnon, qui refuse qu'elle sorte en son absence et l'enferme même parfois pour la faire rester au foyer. Symbole pour elle de ses efforts pour «faire famille», le sapin a sans doute été pour ce compagnon celui de l'insubordination de Nathalie et de son émancipation, déjà amorcée par le déménagement. Face à l'irrationalité, pour elle flagrante, du comportement de l'homme, face à l'anéantissement de sa démarche pour animer positivement son foyer et tenter d'en faire un lieu agréable et convivial, elle abandonne tout espoir de réussir à «discuter» avec son compagnon, de comprendre sa violence et de pouvoir y mettre fin. Cet instant marque la fin de l'empathie manifestée à l'égard de son conjoint, empathie qui lui avait jusqu'alors permis de supporter l'insupportable. Il sonne la fin de l'illusion que les problèmes venaient de la belle-famille et que, une fois seuls dans une nouvelle maison, elle pourrait enfin avoir un foyer «normal». Etre frappée avec le symbole même de cette espérance, le sapin de Noël, c'est trop.

\footnotetext{
${ }^{1}$ Je n'ai jamais trouvé la possibilité de discuter (pour expliquer pourquoi) je voulais partir. J'en avais marre, j'en avais marre. En 2005, j'ai reçu la dernière volée, avec un sapin. C'était Noël, j'avais acheté un petit sapin pour décorer la maison. Il ne me donnait pas d'argent, mais j'avais trouvé un petit boulot au noir. J'ai toujours astiqué la maison, je l'ai toujours faite belle (parce que j'espérais pouvoir) un jour inviter sa famille, la famille de JM, et peut-être même que ma famille pourrait venir, qui sait? Mais, quand même, pour la première fois depuis que j'étais en Martinique, je veux faire que ma maison soit belle pour fêter Noël. Et j'ai reçu une belle volée (avec le sapin...), je sais même pas pourquoi...
} 
Le déclic se caractérise par sa soudaineté et son caractère imprévisible : il surprend tout à la fois l'agresseur et la victime elle-même, qui refuse tout à coup des comportements qu'elle acceptait jusqu'alors. Il peut résulter de l'ultime dégradation et de l'anéantissement des efforts de la femme, comme pour Nathalie. Mais il peut aussi être provoqué par la révolte devant une interdiction, là encore inexplicable pour la femme, d'exercer ce qui lui semble un droit légitime, celui de se livrer à une activité normale, respectable - comme aller chanter à l'église, dans le cas de Delphine :

J'étais dans la terre, en train de jardiner, planter mes persils, mes céleris, tout ça, je jardinais. Je jardinais et quand j'ai fini de faire, je lui dis: 'Bon, je me prépare, je dois, j'ai répétition de chant à..., il faut $y$ aller, et tout'. Parce qu'au départ, il ne voulait pas que j'y aille, et je lui ai dit: 'Mais, bon, pourquoi tu ne veux pas que j'y aille?' et, ce beau jour, il a dit: 'Non, tu ne vas pas, je t'ai dit, tu ne vas pas!'. Et là, je me suis dit : c'est trop, c'est trop. (...) Alors je suis partie, je suis sortie du potager, je suis allée me préparer. Quand j'ai fini de me préparer, il a pris la clé de la voiture. (...) Je lui ai dit: 'Bon, ben, tu as pris la clé de la voiture, et bien garde-la, moi je m'en vais !'. Et puis je suis partie. (...) A ce moment, je suis rentrée chez ses parents, et puis j'ai dit à ses parents ce qui s'est passé. Et c'est à partir de ça, tout ça s'est déclenché. C'est à partir de là qu'il y a eu tout, tout s'est déclenché à ce moment-là. C'est parce que je me suis, j'ai dit: 'Non, ça suffit là maintenant! Je ne peux rien faire... Non, non, non!'. Je me suis dit: 'Non !'.

Contrairement à l'exemple précédent qui se situait dans un contexte festif, l'incident décrit par Delphine se déroule après un labeur qui contribue à alimenter le foyer ${ }^{3}$. Ce n'est que lorsqu'elle a fini sa tâche qu'elle se propose de se rendre à la chorale religieuse. L'interdiction posée par le conjoint lui apparaît d'autant plus exorbitante qu'il lui refuse non seulement le droit de se détendre, mais la prive d'un lien social important pour elle - et

\footnotetext{
${ }^{2}$ Souligné par nous.

${ }^{3}$ Ce qui n'est pas anodin quand on sait que les revenus de cette jeune femme sont entièrement accaparés par son conjoint et qu'elle n'a aucune maîtrise des dépenses du foyer, y compris des dépenses alimentaires. Il nous semble que le jardin potager représente alors un espace de résistance - dont elle peut gérer elle-même les produits obtenus à la force de son travail : c'est elle qui plante les produits et qui s'en sert pour cuisiner.
} 
même de la réalisation d'une aspiration ancienne et profonde, constitutive même de son identitét.

En fait, tout comme dans le cas de Nathalie, l'exaspération à l'origine du déclic résulte de l'incompréhension de l'attitude de l'homme. Pourquoi veut-il l'empêcher de sortir ? Pourquoi le compagnon de Nathalie a-t-il détruit le décor de Noël et s'en est-il même servi pour la frapper ? En fin de compte, pourquoi ces hommes rejettent-ils ce qu'il peut y avoir de positif dans et pour leur femme ? Il y a un instant où l'incompréhension de la réaction masculine permet à la femme de déceler la malveillance qui la fonde, et ce qui apparaît comme le souci premier de l'homme : maintenir son pouvoir absolu sur sa compagne. «J'ai compris tout ton manège », dit alors Delphine. Cette découverte, qui la frappe de son évidence, agit comme un déclic. Elle se précipite ainsi chez ses beaux-parents pour «tout leur dire». Il s'ensuivra une réunion provoquée par plusieurs membres de la famille et des amis proches, où son mari sera mis en accusation, les proches prenant le parti de Delphine, qui, pour la première fois et devant «tout le monde », «parle de tout»: insultes, interdictions, confiscation des ressources, viols conjugaux, etc.

Dans ce cas précis, le hasard qui a fait de Delphine l'une des « cibles » appelées par l'enquête téléphonique, peu après cette confrontation, a joué un rôle de confirmation du bien-fondé du déclic et de sa démarche :

Après, [il y a eu] l'appel [téléphonique]... Et c'est à partir de ça que je me suis dit, comme quoi, tout cö̈ncide... Parce qu'il y a eu ça, après il y a eu l'appel, que j'ai pu parler de tout ça. Je me suis dit: 'Mais comment se fait-il qu'ils m'ont appelée, moi ? J'avais jamais appelé'. Brusquement, il y a ça, on m'appelle, je ne comprends pas, en fait, je ne comprenais rien... Je me suis dit: tout est en train de se dérouler, tout est en train...

Après le déclic, provoqué par un insupportable sentiment de ras-lebol, qui entraîne une réaction immédiate, balayant les hésitations et les inquiétudes à propos de l'avenir, le processus se met, en effet, en marche, et rien ne peut plus être comme avant. Il s'agit souvent d'un choix rapide, décisif pour la dignité et parfois pour la survie. Si ce mécanisme apparaît

\footnotetext{
${ }^{4}$ Aspiration que ses parents adoptifs lui avaient déjà refusé de réaliser dans son enfance : « J'ai fait ma profession de foi très tôt et quand j'ai demandé à servir à l'église, ils m'ont dit 'non', ils m'ont dit 'non, il y a autre chose à faire' [les aider au magasin]. Mais, comme on dit, 'on perd jamais sa route'. La destinée est toujours là... ».

${ }^{5}$ Souligné par nous.
} 
souvent tardif à l'observateur («Une amie, elle se moquait de moi : 'Tu t'es réveillée ?' », raconte Delphine), il restaure la dignité de la victime et signe le début d'une maîtrise nouvelle de sa vie.

Si Delphine espère néanmoins pouvoir rester avec son mari, qu'elle juge «apaisé » après sa mise en accusation publique, au moins jusqu'à ce que leurs enfants aient quitté le foyer, le déclic se matérialise généralement par le départ, rapide sinon immédiat, de la femme. Quittant de sa propre initiative le territoire du conjoint, elle accepte, ce faisant, de s'exposer à des difficultés matérielles, psychologiques, sociales et familiales importantes, et sait que son geste sera lourd de conséquences, comme le rapporte Nicole :

\begin{abstract}
Il y avait une tension qui a duré trois jours, et le troisième jour, les coups sont partis... Je déteste la violence, sous toutes ses formes, mais je ne peux pas rester passive devant quelqu'un qui est violent si je peux me défendre. Donc je me suis défendue et puis... les policiers sont intervenus parce que les voisins, ça avait dépassé les bornes, les voisins ont appelé la police et on l'a emmené. [Elle porte plainte car] j'avais l'intention de ne pas supporter ça, parce que c'était plus que je ne pouvais supporter. Donc c'est la goutte d'eau qui a fait renverser la vasque. Je suis partie le soir même, en pesant le pour et le contre des conséquences que ça allait avoir, parce que des conséquences ça en a, ça en a! Depuis le jour où j'ai laissé sa maison jusqu'à aujourd'hui où je vous parle, il y a des conséquences... ${ }^{6}$
\end{abstract}

Ces conséquences, et les immenses difficultés financières dans lesquelles elle se débat, Manuela en parle longuement. Le père de son fils, chez qui elle vivait, avait «quelqu'un à l'extérieur», ce qu'elle a supporté un temps :

Vous savez, quand on n'a nulle part où aller...

C'est plutôt ça qui vous retient...

Mais, lorsqu'il la frappe, à Noël, parce qu'elle lui reproche ses infidélités, elle part néanmoins «sur un coup de tête » avec son fils, alors âgé de trois ans et retourne chez ses parents, qui l'accueillent fort mal (« Ça a fait tout une histoire. Ils ont dit que c'est moi qui avais fait quelque chose ») et chez qui elle ne reste que le temps de trouver, rapidement, un

\footnotetext{
${ }^{6}$ Souligné par nous.
} 
logement et un travail, pénible, et qui ne lui permet que difficilement de survivre.

Le déclic sanctionne et accélère la prise de conscience par les femmes du fait qu'elles sont victimes de violences. S'il se caractérise par son instantanéité, sa soudaineté et son évidence, et survient, dans la plupart des cas rencontrés dans cette enquête, à la suite d'un incident dans le couple, la prise de conscience, qui se construit patiemment, dans un cheminement pénible jalonné d'incertitudes et de doutes, peut être déclenchée par une intervention extérieure.

Emmanuelle mentionne ainsi la parole d'une amie qui, l'informant qu'elle est «victime de violence psychologique », lui rend la situation plus intelligible. Le banquier, qui ne peut passer outre à l'interdiction du mari de Kelly de lui accorder les fonds qu'elle sollicite, l'incite à lire un ouvrage sur le harcèlement moral, qui, dit-il, l'aidera à comprendre ce qu'il ne peut pas lui expliquer. Cette lecture lui permettra en effet de décoder sa situation et de sortir de sa «surdité » :

C'est là que j'ai commencé à comprendre. Et j'ai commencé à faire attention à tous les détails, car comme vous êtes dans une sorte de nuage, là, comme quelqu'un de sourd en permanence, puisque moralement vous êtes atteint. J'ai commencé à tout analyser, noter...

Des professionnels peuvent aussi attirer l'attention de la femme sur l'anormalité du comportement de l'homme. Dans le parcours de certaines, un épisode de dépression peut ainsi s'avérer salvateur en les obligeant à se questionner sur les sources de leur malaise et à remettre en cause à la fois leur comportement et celui, toxique, du conjoint. Ainsi, Jeanne explique-telle :

Après ma dépression, j'étais prise en main,
psychothérapie, tout ça. Donc, j'ai fait un travail
sur moi. Un travail de valorisation, de
réassurance, je me suis mise en avant, en tant que
personne, ce que je n'avais jamais fait. On m'a
tellement répété que j'étais rien, donc maintenant
que mes yeux se sont ouverts, je me suis dit: 'C'est
pas normal que je subisse ça...'.

La peur de la solitude retient cependant encore la jeune femme, qui, s'étant «construite » en métropole, dès sa sortie du lycée, loin des siens et dans une relation fusionnelle avec son conjoint, ne peut plus envisager sa vie sans celui qu'elle considère comme "sa béquille». Elle se révolte même contre l'équipe soignante qui, ayant analysé le comportement de son 
compagnon, la met au défi de lui dire en face qu'il est la cause de sa dépression, ce qu'elle nie et refuse vigoureusement, par peur des représailles et bien qu'elle « sente que c'était le moment». Elle restera donc encore « un an à bricoler comme ça ».

La prise de conscience suppose nécessairement une conscience de soi et de ses propres désirs. Or, la femme victime de violences, qui vit sa situation «dans un nuage », qui se sent dévalorisée, qui, pour la paix du ménage, a parfois choisi de «se rabaisser », de ne pas «se mettre en avant», en renonçant à sa personnalité, à son autonomie, à sa vie sociale, ne peut aisément investir son existence propre, interroger ses désirs : «Je n'existais pas », dit Jeanne. La parole d'autrui peut ainsi s'avérer libératrice, les rencontres fortuites également, lorsqu'elles renseignent sur soi-même. Jeanne, par exemple, explique avoir commencé à comprendre l'anormalité de sa situation après un bref flirt avec un homme qui la traite bien mieux que son conjoint. Mais la réflexion ainsi entamée a été bien longue à produire ses fruits : ce n'est que quatre ans après ce « petit coup d'air » et une dépression grave qu'elle se décidera à partir. L'histoire de cette femme illustre bien comment la sortie de la situation de violence se construit dans une temporalité saccadée, marquée par des épisodes de lucidité saisissants étouffés par la peur, ou par l'espoir, suivis de phases d'acceptation plus ou moins passive pour finalement aboutir à l'incident qui servira d'interrupteur, de déclic, puis de catalyseur. Lorsque son compagnon décide de revenir vivre en Martinique, ce qu'elle ne souhaite pas pour elle-même, Jeanne envisage ainsi de ne pas le suivre et de mettre fin à leur relation :
[Je sentais que si j'acceptais de partir avec lui]
C'est comme si je me mariais. Je ne sais pas. C'est comme si je faisais un engagement, comme si je m'engageais pour la vie avec lui. J'ai dit: 'C'est là le moment ou jamais de dire: Stop'. (...) Je me suis sentie forte pendant les trois premiers mois de son départ, et puis j'ai craqué à la fin.

Elle le rejoint donc en Martinique, en pensant à ses enfants, encore jeunes. Elle ne partira finalement, «hors d'elle» (hors de la femme soumise qu'elle a été pendant plus de quinze ans), que lorsqu'elle sentira sa vie menacée :

Je voulais attendre leur majorité pour faire ce paslà, mais quand j'ai vu la fureur qu'il y avait dans les yeux de M. la dernière fois où il m'a tapé dessus, la haine, la violence, le désir de meurtre, je ne sais pas, d'en finir avec moi, je me suis dit que peut-être je n'ai pas le temps d'aller jusque-là. 
Alors je suis partie avec mon petit bagage à main, mes bigoudis sur la tête, et advienne que pourra!

Si le déclic peut se produire au terme d'une lente et progressive prise de conscience, il fonctionne en effet comme une révélation, éclairant brutalement la situation sous un jour nouveau, induisant une action immédiate et écartant de ce fait la peur qui retenait jusqu'alors la femme quand ce n'est pas cette même peur, devenue extrême, qui, comme dans le cas de Jeanne, constitue justement le déclic salvateur. La femme se réapproprie alors sa propre existence, en prenant en compte ses désirs profonds et en écartant toute autre préoccupation qu'elle-même (y compris celle des enfants quand ceux-ci ne sont pas menacés par le père) et le souci de sa survie. Ceci implique donc une déculpabilisation, même brève, mais suffisante pour, comme Jeanne, franchir le pas :

Pour une fois, penser à moi. (...) Comment dire, ce n'est pas un acte d'égoüsme de se séparer de... à ses dires, de 'la personne qui vous a donné tous son amour pendant vingt ans' - c'est comme ça qu'il définit les choses. Je n'ai pas à culpabiliser, j'ai simplement voulu me sortir d'une situation, parce que je suis responsable de deux êtres humains à qui je dois une part de bonheur, en premier lieu, et à moi-même, en deuxième lieu.

Momentanément mise à distance, la culpabilité ne disparaît toutefois pas du simple fait du départ et peut se maintenir insidieusement, enfermant la femme dans un doute perturbant sa reconstruction. La famille de Jeanne, «dégoûtée » d'elle, pense qu'elle aurait pu «supporter plus », qu'elle a « fait tout un tapage pour rien», car elle n'était pas «plus malheureuse qu'une autre ». Jeanne elle-même, qui bénéficie pourtant d'un suivi thérapeutique, se persuade parfois qu'elle aurait pu, comme sa mère avant elle :

Supporter, voilà, c'est exactement ça. J'aurais pu supporter. Il suffisait d'attendre que les choses aillent mieux pour lui, que la vie lui sourie, qu'il soit mieux dans sa tête.

Et ce d'autant que son ex-compagnon lui «offre une image, actuellement, de quelqu'un de tellement sympa », qu'elle se dit: «Mince alors ! Je suis passée à côté de quelque chose. Il est capable de changer ». C'est son père, auteur autrefois de violences extrêmes à l'encontre de la mère de Jeanne, qui, ayant fait «beaucoup de chemin sur lui » avec son retour à la religion, la soutient, car «il sait quel est le cheminement d'un homme violent », et lui dit «fermement : 'Tu ne retournes pas avec lui !' ». 


\section{II. - LE DIFFICILE RENONCEMENT À LA RESPECTABILITE, À L'IDÉAL CONJUGAL OU AMOUREUX}

Pour un certain nombre des femmes rencontrées, le déclic ne s'est pas encore produit. Bien qu'elles aient répondu positivement, lors de l'enquête téléphonique, à des questions portant sur les faits de violences subis dans le cadre des relations conjugales ou amoureuses (Vanessa et Alexie : insultes et menaces de mort, verbales ou avec arme), ou qu'elles se soient adressées à l'Union des Femmes de la Martinique (Anne et Thérèse), elles ne se reconnaissent pas victimes de violences conjugales ou, comme Vanessa, préfèrent «ne pas le savoir ».

Si le renoncement - à son indépendance économique, à sa vie sociale, à ses désirs, à sa personnalité - fonde largement la situation des femmes victimes et leur permet de la «supporter ${ }^{7}$, c'est aussi, à l'inverse, la très grande difficulté qu'il y a à renoncer à l'espoir d'une amélioration de la situation, mais, plus encore, à ses valeurs et à ses idéaux - la respectabilité, l'image idéalisée du couple et de la famille, l'amour - qui retarde la prise de conscience, la production du déclic et la sortie de la situation.

Vanessa et Alexie ne peuvent ainsi accepter l'échec de la relation avec leur conjoint ou partenaire, si insupportable et source de dépression soit-elle pour elles, car elles ne peuvent admettre d'avoir, comme leur mère, plusieurs hommes dans leur vie (Vanessa) ou ne peuvent (encore) abandonner l'espoir que le père de leurs enfants cesse d'avoir d'autres femmes (et des enfants avec elles) et qu'il les choisisse finalement et définitivement, elles seules (et leurs enfants). Même si, comme Alexie, elles sont bien conscientes qu'elles «ont toujours eu des projets» conjugaux, «mais pas lui ».

Comme Alexie et Vanessa, Stéphanie n'arrive pas à faire le deuil de sa première - et, à ce jour, unique - relation amoureuse. Relation dont, elle aussi jeune et sans expérience, elle est également «tombée » rapidement enceinte d'un enfant mal accepté par son partenaire. Son compagnon l'isole des membres de sa famille et de ses amis, auxquels il interdit l'accès à sa maison. Il la laisse sans ressources personnelles bien qu'elle s'occupe de sa comptabilité et du ménage, se montre «très dur» avec elle et sort avec d'autres femmes. Il lui dit qu'elle est «une grosse charge» et "l'embarrasse » chez lui, et, tour à tour, lui intime de partir et l'empêche de le faire. Elle se sent «très mal », blessée, déprimée, «fatiguée de tout ça », sans courage. Elle voudrait «ne plus dépendre de lui », «s'en sortir» et

\footnotetext{
${ }^{7}$ Voir, dans ce numéro : «'Pourquoi ne partent-elles pas ?' Les obstacles à la sortie de la situation de violence conjugale. ».
} 
avoir son «chez elle». Mais, il ne lui est «jamais venu à l'esprit» de contacter des associations ou des structures d'aide sociale, car elle non plus ne se reconnaît pas victime de violence conjugale :

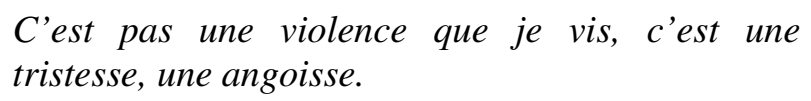

Elle sent néanmoins que la relation a «atteint ses limites». Son compagnon semble ne plus tenir à elle : il voudrait » une femme élégante, qui a beaucoup d'argent, qui aurait un emploi et pourrait lui offrir tout ce qu'il veut » et il n'accepterait, dit-elle, de l'épouser que si elle lui apportait suffisamment d'argent. Mais, elle est «sans le sou», ayant abandonné sa propre mini-entreprise pour vivre avec lui et s'occuper de sa maison et de leur enfant. Elle se sent trop déprimée pour être capable d'entreprendre une autre activité, d'autant que, sans argent, sans permis de conduire, vivant loin de Fort-de-France sur le terrain familial de son compagnon, elle se sent démunie, «perdue », impuissante. Elle estime à la fois qu'il y a « urgence » à mettre fin à cette relation et qu'il (n') y aurait « véritablement urgence » à partir (que) s'il la frappait. Là serait, dit-elle, «la goutte d'eau qui ferait déborder le vase » et qui l'obligerait à «prendre ses cliques et ses claques » et à s'en aller. Mais elle craint aussi que ce soit justement son départ, ou l'annonce de son départ, qui déclenche la violence physique de son compagnon. Elle est en effet persuadée qu'il «n'acceptera pas qu'elle parte avec des affaires », mais il lui semble trop injuste de devoir partir sans rien emporter et « repartir à zéro, à zéro, zéro, total zéro ». Il lui est insupportable de partir les mains vides, mais, plus encore, de constater l'échec complet de sa première et seule relation de couple, de ses efforts pour «créer un foyer », le manque d'amour de son compagnon pour elle, ainsi que son absence d'intérêt pour leur enfant, dont il ne voulait pas :

Il m'a donné un enfant et puis voilà. Et puis, c'est tout. Ça s'arrête là...

Mais, elle n'a pas (encore) réellement renoncé à cette relation : elle prévoit que les choses vont être «encore plus difficiles », mais espère que, si elle trouve le courage et les moyens de partir, il (la ?) «comprendra » enfin.

Si Alexie et Vanessa ne peuvent faire le deuil, trop douloureux, d'une relation émaillée de violences - ne serait-ce que celle du «silence» gardé par le compagnon de Vanessa et qui, pour elle, nie jusqu'à son existence -, si Stéphanie ne se sent pas le courage de partir seule avec son enfant sans rien emporter et sur un constat d'échec, c'est à la volonté de « guérir » l'autre qu'Anne et Thérèse n'arrivent pas à renoncer.

Quand nous les avons rencontrées, Anne et Thérèse ne vivaient pas avec le conjoint auteur de violences, la première, parce que, à bout de forces psychologiques, elle s'était récemment résolue à le quitter, la seconde, parce 
qu'il était incarcéré, pour la troisième fois, pour violences conjugales. Ces deux femmes, que nous avons rencontrées à l'UFM ${ }^{8}$, ne s'y étaient pour autant pas adressées pour se plaindre des violences subies, mais pour y chercher des conseils pour "guérir» leur compagnon de son alcoolisme, voire du sort jeté sur lui et qui serait responsable de sa violence. Contrairement à Vanessa, Alexie et Stéphanie, leur partenaire n'était pas le premier et elles n'étaient pas jeunes et inexpérimentées quand elles l'avaient rencontré.

Anne, née en Europe du sud, a accepté l'entretien «pour aider la recherche », tout en affirmant qu'elle n'était pas une «victime de violences conjugales ». Arrivée de l'hexagone il y a quatre ans, sans enfant, elle éprouve alors, analyse-t-elle maintenant, le «besoin de tomber amoureuse » et, bien qu'elle sente qu'il y a « quelque chose qui cloche» chez cet homme «charmant», commence une liaison avec le propriétaire martiniquais du «bas de villa » qu'elle loue. Elle espère que l'amour pourra les guérir, lui, de son alcoolisme et des souffrances nées d'un » lourd secret de famille», elle, de son enfance douloureusement marquée par l'émigration lorsqu'elle avait huit ans et le suicide de sa mère lorsqu'elle en avait seize. Mais, après quatre ans de vie commune, jalonnée de séparations (elle regagne alors le rez-de-chaussée de la maison dont son ami est propriétaire et dont il occupe l'étage), de brèves périodes «sans alcool » et de longues périodes «avec », elle « craque » :

On devait partir ensemble en vacances au mois d'août, on avait les billets, on avait tout. Il est rentré à trois heures du matin, puant l'alcool à plein nez. Je travaillais le lendemain. J'ai appelé ma souur en métropole et je lui ai dit: 'Je m'en vais, je n'en peux plus!' et je suis partie au boulot avec mon sac dans la voiture, et j'ai complètement craqué, j'ai complètement craqué, je suis allée dans la salle de pause, je ne pouvais plus... Mon patron m'a emmenée voir sa femme qui est psychologue, le jour même, et je suis restée [avec elle] tout l'après-midi (...) et je suis allée chercher mes affaires. (...) Il m'a vue, donc je lui ai dit: 'Je viens juste chercher mes affaires' - et, là, j'ai revu le gars anéanti, le visage défiguré de douleur, il s'était mis à pleurer et à me dire: 'Mais, pourquoi j'arrive pas? Pourquoi j'arrive pas?'. Et, là,

\footnotetext{
${ }^{8}$ Union des femmes de la Martinique.
} 
j'étais détendue et je lui ai dit que: 'Non, on n'y arrive pas, on n'y arrive pas', et puis je suis partie.

Mais, au retour des vacances, il veut la revoir. Elle prend rendezvous pour lui chez une psychologue, "parce que, même si c'est fini entre nous, je voudrais qu'il entende certaines choses ». Il contacte une association anti-alcoolique. Mais, au bout de deux mois, il «craque», lui aussi, de nouveau :

Au bout du troisième mois, un jour, il est arrivé, et puis j'ai tout de suite vu qu'il sentait l'alcool. C'était juste une bière. Mais, je me suis dit: 'Ça va repartir...'. Et, en plus, quand je lui ai dit: 'Tu as bu?' - 'Oh, une bière!' Il s'énerve, ça recommençait. Donc, je me suis dit: 'Il faut partir' et je suis partie...

Le départ ne suffit pas à mettre fin à la dépendance - ni alcoolique ni amoureuse. Elle déménage et lui part en métropole pour des raisons de santé, mais :

J'y pensais tous les jours, à regretter tout ce que j'ai vécu, la honte, les humiliations, les machins, une boule, là. Et j'arrivais petit à petit à reprendre et, pouf, ça repartait, quoi. J'étais affaiblie, blessée, ça a été hyper-dur. (...) Comme je vous le disais, j'ai besoin de comprendre, parce qu'on ne sait pas quoi penser, on oscille entre la paix et... L'autre jour, j'étais à deux doigts de l'appeler et de lui dire: 'On reprend', en me disant: 'Je vais lui dire tout ce qu'il m'a fait! Mais, non, je vais le revoir [et je vais avoir] envie de le prendre dans mes bras !'. Et puis, y'a d'autres moments où... Et ça c'est vachement déstabilisant, on a honte d'être comme ça, on a honte.

Pas encore «reprise », au moment de l'entretien, Anne reste très «reprenable ». Thérèse, beaucoup moins à l'aise avec le français ${ }^{9}$ et avec la verbalisation des sentiments et des affres du cœur, l'est encore bien davantage. Elle n'aime pas la violence et n'avait jamais été battue, dit-elle, ni par ses parents, ni par les pères de ses premiers enfants. Le problème avec ce dernier compagnon, incarcéré plusieurs fois pour violences conjugales mais qu'elle aime beaucoup, c'est aussi, selon elle, l'alcool :

\footnotetext{
${ }^{9}$ De père haïtien et de mère dominicaine, elle a vécu au Venezuela ; pendant l'entretien, elle s'est exprimée le plus souvent dans un mélange d'espagnol et de créole haïtien.
} 
C'est le rhum et la fête, l'alcool, l'alcool, le whisky, tout ça, avec des camarades aussi.

Jaloux, il l'enferme quand il part, lui confisque son portable, la suit partout et ne la laisse pas aller seule aux toilettes dans les lieux publics. Il la met aussi dehors, parfois nue ; elle se réfugie alors chez une copine qui lui prête des vêtements pour qu'elle puisse aller à la police, mais elle n'aime pas « rester chez les gens »:

\begin{abstract}
Alors, j'ai des problèmes avec la copine, et chaque fois que je veux bouger, je retourne avec lui. Moi, je l'aime beaucoup, mais, quand il me frappe, ça m'a énervée, je n'aime pas ça, si on est ensemble, ça peut pas aller avec la violence, je n'aime pas la violence.
\end{abstract}

$\mathrm{Au}$ moment de l'entretien, elle ne vit pas avec lui, puisqu'il est incarcéré. Elle se démène, pour trouver du travail, mais, surtout, pour essayer de récupérer les trois enfants qu'elle a eus de son conjoint précédent et qui sont placés - et pour éviter que l'on place la petite fille née de son conjoint actuel et l'enfant qu'elle attend de lui. Elle contacte l'assistante sociale, le CCAS $^{10}, l^{\prime}$ UFM, qui adresse des courriers à la gendarmerie, à la Préfecture, etc. Mais elle pense que si on la menace de lui retirer ses enfants, c'est par jalousie - ou parce qu'on lui a jeté un sort. Evangéliste, elle envisage d'ailleurs d'abandonner cette Eglise, qui était celle de sa mère, pour exploiter, comme son père, " séancier vaudou », son don de vision - et, peutêtre, «agir» sur les services sociaux afin qu' on ne lui retire pas ses enfants. Mais, à la question de l'enquêtrice : «Là, vous êtes sûre de ne pas retourner avec votre ex-compagnon?», elle répond simplement et franchement: «Non ».

\title{
III. - LE RECOURS AUX INSTITUTIONS
}

Les femmes que nous avons rencontrées par l'intermédiaire de l'Union des Femmes de la Martinique ou du Centre Rosannie Soleil ont, par définition, effectué elles-mêmes des démarches auprès des institutions spécialisées dans l'aide aux femmes victimes ou ont été dirigées vers elles par des travailleurs sociaux. Elles ne vivent plus avec le conjoint ou ami auteur des violences rapportées et, à l'exception d'Anne et de Thérèse, considèrent la relation comme terminée - même si Jeanne se demande si «peut-être » elle ne pourrait pas revivre un jour avec le père de ses enfants.

\footnotetext{
${ }^{10}$ Centre communal d'action sociale.
} 
Cinq femmes, qui avaient besoin d'un logement pour fuir le conjoint violent, ont intégré le CHRS $^{11}$, le CENDRA ${ }^{12}$ ou un autre hébergement social : Henriette, Nathalie, Nicole, Christelle et Jeanne. Seule cette dernière, une Martiniquaise cadre moyen qui a vécu longtemps dans l'hexagone, a pris directement contact avec l'UFM, association qui travaille en partenariat direct avec les différents centres d'hébergement et qui l'a orientée vers l'un d'eux. Les quatre autres femmes ont pu le faire grâce à l'intervention d'une assistante sociale qui les a orientées vers l'UFM, et a constitué leur dossier d'admission en centre d'hébergement. Mais partir ne leur a pas été facile. D'autant que, venues des états voisins de la Caraïbe, elles étaient d'origine étrangère, en situation précaire et sans famille en Martinique.

\section{A. - Partir ? Fuir ? Mais où aller ? "}

Nathalie: J'ai pas de famille. J'ai tourné rond dans direction Martinique pour voir où ça je peux aller. J'étais mal.

Même lorsqu'on a pris conscience de l'anormalité de sa situation et de la nécessité d'en sortir, quitter un conjoint violent n'est pas aisé. On se heurte d'abord à la nécessité - et à la difficulté - de trouver un nouveau logement. L'absence fréquente de revenus salariaux (deux femmes seulement sur les huit rencontrées par l'intermédiaire des institutions d'aide avaient une profession et un emploi qui leur conféraient une certaine autonomie financière) ne permet pas de recourir au secteur locatif privé - et augure mal de la survie matérielle après le départ, surtout lorsqu'il y a des enfants à charge.

Pour les femmes d'origine étrangère, particulièrement, et surtout lorsqu'elles ont été enfermées, l'absence de papiers d'identité ou de séjour, la méconnaissance de leurs droits et des structures d'aide, l'incompréhension ou la négligence des travailleurs sociaux, l'insuffisante maîtrise de la langue française, l'absence de soutien familial ou de réseau amical, représentent autant d'obstacles. La surface limitée du pays et l'insularité apparaissent aussi comme des freins majeurs lorsqu'il s'agit de quitter le conjoint ou de

\footnotetext{
${ }^{11}$ Centre d'hébergement et de réadaptation sociale, géré par l'association Rosannie Soleil.

${ }^{12}$ Centre Départemental de Ressourcement et d'Accompagnement dans la vie, acronyme faisant écho à «l'affaire Sandra» (jeune femme arrosée d'essence et brûlée vive par un partenaire qui n'acceptait pas la séparation).
} 
rechercher de l'aide: l'impossibilité de se cacher en Martinique ${ }^{13}$, l'impossibilité, même, que les démarches entreprises restent confidentielles, sont souvent évoquées. Il faut compter également, bien sûr, avec la crainte des représailles du conjoint, la peur d'être tuée. Tout ceci conduit très souvent les femmes à hésiter, à reporter leur décision jusqu'à ce que des solutions se présentent ou à ce que la crainte pour leur vie ou celle de leurs enfants ne permette plus les atermoiements.

Henriette, d'origine dominicaise, est ainsi restée vingt-sept ans avec un mari «sauvage » et violent. Elle ne l'a quitté que lorsqu'il l'a poursuivie «avec un grand couteau » parce qu'elle refusait d'avoir des rapports sexuels avec lui, le sachant contaminé par le virus du sida, et (peut-être surtout) parce qu'il a menacé de tuer leur fils qui avait pris sa défense (menace que le père tentera apparemment de mettre à exécution par la suite). Elle va alors voir les gendarmes, qui lui conseillent de contacter une assistante sociale, laquelle l'oriente vers l'UFM. Elle explique pourquoi elle n'est pas partie plus tôt :

Je ne travaillais pas. J'étais mal informée. On ne connaissait pas l'Union des Femmes. Qui allait nous apporter une aide? [Vous n'aviez pas d'amis? de connaissances? de famille du tout, ici ?] Non, non, pas vraiment, parce que je n'aime pas... embêter les gens avec mes problèmes, tu vois. [Il n'y avait pas quelqu'un pour vous aider?] Non, pas vraiment. Personne en fait. Qui me connaissait? Qui je connaissais? Personne! Hein, personne!

Nathalie, également originaire de la Dominique, s'était adressée à une assistante sociale à la suite de violences infligées à son jeune fils par son compagnon, mais s'était vu apporter comme seule aide le conseil de se rendre elle-même à la gendarmerie. Pensant que l'assistante sociale avait partie liée avec la famille de son compagnon et craignant les menaces de celui-ci, elle avait abandonné ses démarches, se résignant provisoirement à son sort.

Nicole, haïtienne, s'est aussi longuement interrogée avant de mettre en cuvre son projet de départ, consécutif aux premières violences conjugales, car, à la peur et aux difficultés matérielles, s'ajoutait la question du titre de séjour :

\footnotetext{
${ }^{13}$ Pendant la séquestration de Christelle, qui a commencé lorsqu'elle était mineure, sa mère avait lancé un avis de recherche. La gendarmerie ne l'a cependant pas retrouvée au cours des trois années où son compagnon l'a retenue dans une masure isolée à la campagne.
} 
Ca a été l'enfer, ça a été l'enfer, et le plus dur c'est que je savais qu'il savait que je n'allais pas prendre ma décision à la légère. Il savait que j'allais mûrement réfléchir avant de faire quoi que ce soit. (...) Donc, c'est pour cela, impérativement, il me fallait le titre de séjour. Il savait très bien que je n'allais pas partir dans un pays où la réalité est que, malheureusement, on peut kidnapper ton enfant, te rançonner, et tuer ton enfant...

Pour Magali, vivant alors dans l'hexagone, partir avec quatre enfants, dont trois en bas-âge, apparaissait quasi impossible. Elle n'a d'ailleurs pu le faire qu'après plusieurs années et une longue préparation :

Une dizaine d'années, une dizaine d'années. (...) J'ai préparé mon départ, parce qu'à plusieurs reprises, je pouvais partir, mais je ne voulais pas partir dans n'importe quelles conditions. (...) Les enfants étaient petits, et j'avais peur de ne pas trouver de garde. Parce que je suis allée visiter quelques amis, et j'ai parlé aussi, et la seule chose qu'ils me disaient, c'était: 'Tu as déjà beaucoup d'enfants' et 'C'est très difficile de faire garder'. (...) Les trois petits, c'était très difficile à pouvoir gérer pour pouvoir dire: 'On s'en va', pour pouvoir partir sur de bonnes bases. A ce moment, j'ai dit: 'Je vais attendre que les enfants aient quatre-cinq ans, et préparer des documents, préparer tout ce que je vis au quotidien, pour pouvoir entamer une procédure'.

Parfois, comme dans les deux derniers cas évoqués, la rupture est un long processus et le départ a été préparé, de plus ou moins longue date. Mais, parfois, il n'est plus possible d'attendre. La fuite apparait comme la seule solution possible pour protéger les enfants et/ou pour sauver sa vie.

Au moment de l'entretien, Christelle, qui avait été adressée à l'UFM par une assistante sociale, venait d'être admise au CHRS. Mais elle avait connu auparavant « une vraie galère ». Interdite de sortie par son compagnon depuis la naissance de son fils, elle avait réussi, quand l'enfant a eu trois ans et est allé à la maternelle, à obtenir de l'ANPE une formation en entreprise que son compagnon avait acceptée, de mauvais gré. Mais elle se rend compte au bout d'un certain temps, qu'au lieu d'aller faire ses djobs, il s'installe en face du lieu où elle-même suit sa formation, pour l'espionner. La relation se dégrade et s'aggrave encore, si cela est possible. Elle ne veut plus avoir de rapports sexuels. Il la force. Elle découvre qu'il est marié et 
qu'il a déjà quatre enfants de son épouse, sans compter d'autres enfants « dehors ». Au moment du carnaval, elle sort pour la première fois, et sans lui, avec des copines. Il porte alors plainte à la police pour abandon d'enfant, menace de la dénoncer comme «sans papiers» - alors que c'est vraisemblablement en raison des plaintes qu'il dépose contre elle et de ses interventions auprès de la Mairie et de la Préfecture qu'elle n'arrive pas à obtenir le récépissé nécessaire pour avoir ses papiers et pouvoir passer son examen $^{14}$. À la suite de cette sortie, il la frappe violemment :

[Les gendarmes] ne sont pas venus, alors que je suis tombée sur la tête. Alors qu'est-ce que j'ai fait? J'ai décidé de partir, je suis partie... J'ai paniqué : j'ai nulle part où aller, j'ai pas d'amis, ma mère vit avec mon beau-père, je ne suis pas en bons termes, je ne peux pas aller chez elle. J'ai une amie à S., c'est elle seulement. Je l'ai appelée, je lui ai dit que j'ai beaucoup de problèmes en ce moment, est-ce que je peux venir chez elle? Mais, chez elle n'était pas... un endroit pour emmener mon enfant.

Elle décide donc de partir seule dans un premier temps et de chercher un logement pour récupérer son fils après l'année scolaire :

Donc, dans un premier temps, je vais partir, et comme l'année scolaire n'était pas loin d'être terminée, je vais pouvoir le récupérer [à la fin de l'année]. Je suis partie, le cour brisé, comme je savais qu'il était à l'école et que c'était lui qui devait aller le chercher... Mon Dieu, je tremblais, je tremblais... en me disant: 'J'ai laissé mon fils derrière moi', mais il me fallait partir. (...) Tous les soirs, je l'ai appelé, au début, quand je pouvais. Après, durant deux-trois mois, je n'ai plus entendu de nouvelles. A cette époque-là, en mai, en juin, quand je suis partie, j'ai fait une dépression, je suis allée voir un psychologue, un psychiatre qui m'a donné des calmants. Et je lui ai expliqué dans quel contexte je suis partie, la violence, la séquestration et tout, et que je subissais des rapports forcés... Alors, j'ai fait tout ce qu'il

\footnotetext{
${ }^{14}$ Elle découvrira plus tard, que son titre de séjour avait, en réalité, été fait l'année passée (mais ne lui avait pas été remis, sans doute suite aux pressions et plaintes de son conjoint) et qu'elle aurait donc pu passer cet examen.
} 
fallait faire pour pouvoir le récupérer [l'enfant] auprès du juge. Alors, j'ai essayé, en attendant, de trouver un logement, mais comme je ne pouvais pas trouver du travail parce que je n'avais pas de papiers... La dame qui m'embauchait au noir, j'ai continué à y aller pour faire des djobs. Elle me payait vingt euros par mois. (...) Dans un premier temps, je suis restée chez ma copine, et quand ma mère [rencontrée par hasard dans la rue alors qu'elle fuyait elle-même son conjoint violent] est venue, elle a vu là ou je vivais, elle a dit, 'Oh la la, tu ne peux pas rester ici!'... Elle a été voir une copine à elle, une Haütienne, et, comme [celle-ci] avait un enfant et n'avait pas de mari, je suis allée chez elle depuis juin jusqu'au mois de décembre.

\section{B. - L'importance du relais des associations et des assistantes sociales}

Henriette: J'ai vu l'assistante sociale, elle a appelé l'UFM. J'ai été, et puis j'ai passé par le CENDRA, je suis restée deux mois et demi, et puis après j'ai venu au CHRS.

Si le tissu associatif est relativement bien étoffé à la Martinique, il n'en demeure pas moins que nombre de femmes ne sont pas capables de s'y repérer et d'aller y chercher directement de l'aide. Le contact avec l'assistante sociale apparaît souvent comme un préalable nécessaire pour être orientée vers les structures spécialisées dans la prise en charge des victimes de violences conjugales.

Le premier contact avec l'assistante sociale n'a pas forcément pour but de dénoncer les faits de violence. C'est parfois à l'occasion de demandes de secours et d'aides sociales ou de la recherche d'un travail, voire de consultations relatives aux enfants, que les victimes évoquent leur situation conjugale. La rencontre avec une assistante sociale n'est d'ailleurs pas toujours à l'initiative de la femme.

Ainsi, Nathalie, qui avait été rebutée et découragée par sa démarche auprès d'une première assistante sociale de secteur, a la «chance » d'être convoquée plus tard par l'assistante sociale scolaire, au sujet de l'absentéisme de sa fille qui a quitté l'établissement et est repartie en Dominique pour fuir la violence - et, sans doute, les «attentions »- de son beau-père : 
$Y$ a une assistante sociale qui m'a convoquée pour ma fille qui était à l'école ici, j'ai expliqué tout ça, j'ai dit je voulais parler, j'ai des problèmes. Et puis c'est l'assistante sociale, Mme V., c'est une Blanche, elle ne comprend pas bien [mon françaiscréole], mais j'ai dit: 'Je vais parler doucement pour faire des choses entrer, pour voir comment ça je vais m'en sortir'. Et puis elle a appelé l'UFM, elle a vu les coups [que] l'enfant, mon fils a. Elle a dit: 'Si je peux faire plus pour toi, je vais faire plus pour toi'.

\section{C. - L'aide d'une communauté religieuse : Magali}

Avant de solliciter l'aide des services sociaux et des organismes d'insertion, c'est à sa communauté religieuse que Magali a fait appel pour pouvoir partir avec ses enfants. Sa fuite - dramatique ou rocambolesque - a commencé dans l'hexagone, où elle était née et vivait avec son second mari, militaire. Ayant surpris celui-ci, dit-elle, dans une situation que sa religion réprouvait fortement, elle veut divorcer. Elle se heurte aux pressions exercées par la riche et influente famille de son conjoint - dans laquelle, ditelle, « on ne divorce pas » - pour l'empêcher de trouver un logement, des modes de garde, une formation et, sans doute, pour la faire taire (est-ce pour cela que son mari a, dit-elle, tenté de l'étrangler ?) et l'empêcher de nuire à leur réputation en parlant de ce qu'elle a vu. Elle prépare soigneusement sa fuite :

Je faisais tout en cachette, je faisais des cartons en cachette, je disais aux enfants ce que je faisais, de ne pas parler, de faire attention. (...) Il a verrouillé toutes les portes de la maison pour ne pas que nous rentrions, et il nous a vraiment mis à la rue. [On avait prévu de partir le lendemain] et on n'a même pas pu re-rentrer dans la maison pour prendre nos affaires personnelles, parce qu'on avait déjà tout fait, mais on n'avait pas mis dehors encore - puisque je ne prenais aucun meuble, absolument rien, juste des cartons et un peu de vaisselle, des choses dont nous aurions besoin, et tout le reste était resté dans la maison, et, malgré ça, il nous a empêchés de rentrer dans la maison, mais $P$. [son fils aîné] a quand même pu rentrer dans la maison, avec son copain, et on a pu rentrer 
par une fenêtre - tout le voisinage nous regardait - pour pouvoir récupérer tous nos cartons, et nous les avons tous mis dehors. Quelqu'un est venu nous aider à les récupérer, une voisine, et on a fait venir une camionnette pour pouvoir les faire partir en container pour la Martinique.

Le récit de Magali fait ensuite état du kidnapping des enfants par la famille paternelle, et de graves sévices qui leur sont alors infligés. Pour retrouver ses enfants, les localiser et les reprendre, elle cherche et trouve, contre ces «gens assez puissants», et, pense-t-elle, contre leur réseau politique et maçonnique, l'aide de sa communauté religieuse :

\begin{abstract}
Plusieurs papas se sont rassemblés avec moi, et il $y$ avait plusieurs voitures, il y avait une cinquantaine de personnes, et on est allés faire toutes les écoles qui étaient aux extrémités, et y avait deux-trois personnes qui se postaient devant l'école, pour, s'il y avait un problème, qu'on puisse soit repartir, soit repartir avec les enfants. Lorsque l'on a retrouvé les enfants, la directrice, elle m'a dit: 'De toutes façons, vous êtes la mère, vous avez les papiers des enfants, et on vous donne les enfants'. (...) On nous les a donnés sans aucune difficulté. Et nous sommes repartis sur la région parisienne, où on s'est occupés des enfants, où ils ont dormi pendant plusieurs jours.
\end{abstract}

Après avoir vécu cachée assez longtemps dans une ville de province, toujours aidée par sa communauté, elle est arrêtée brutalement par la police et emprisonnée, à la suite d'une plainte déposée par sa belle-famille, qui a retrouvé sa trace, et ses enfants sont placés. Mais, devant la justice, racontet-elle, « la situation s'est renversée ». Cependant, son mari s'étant « enfui » à l'étranger, où, après avoir quitté l'armée, il travaillerait pour une multinationale, elle n'arrive pas à faire prononcer le divorce et à bénéficier d'une pension alimentaire. Pour pouvoir quitter l'hexagone, elle a de nouveau sollicité l'aide de sa communauté. Elle a ainsi pu, grâce à une collecte, payer les billets et rentrer à la Martinique, où une autre communauté a pris le relais pour l'accueillir et l'héberger :

Je suis arrivée en janvier... Mais je suis arrivée sans argent, sans rien. C'est-à--dire que c'est ma communauté qui a payé mon billet d'avion pour que je vienne ici en Martinique et une ici a continué à m'aider. 
Reconnaissante à ses coreligionnaires de l'aide matérielle qu'ils lui ont apportée, elle s'est quand même sentie très seule face à la justice :

J'avais des vêtements, j'avais de l'alimentation, mais j'ai quand même été seule, face à tout, parce qu'ils ne pouvaient pas me suivre juridiquement et c'était dans ce domaine-là que j'avais le plus besoin d'aide.

C'est d'ailleurs pour trouver cette aide qu'elle s'est adressée à l'UFM, où nous l'avons rencontrée. Sans ressources, elle bénéficie de l'aide juridictionnelle, mais a le sentiment de n'être pas bien défendue, croyant même que sa belle-famille a réussi à corrompre l'avocate qu'on lui a désignée. Jusqu'à ce qu'un nouvel avocat, persuadé «qu'il y a de la corruption dans le dossier », n'accepte, dit-elle, de s'occuper «pratiquement gratuitement » de son cas. Outre l'assistance de sa communauté, elle a donc trouvé en Martinique celle de l'UFM, mais elle estime ne pas recevoir l'aide qu'elle serait en mesure d'attendre de la part de «l'antenne d'insertion, ni tous ces organismes $»$ :

On arrive avec des dossiers pratiquement bouclés, et puis on passe son temps à attendre... L'agence d'insertion, j'y suis inscrite depuis trois ans, et, depuis, le dossier n'a pas avancé...

\section{IV. - SE RÉSIGNER À LA SÉPARATION}

\section{A. - Se rendre indifférente pour se préparer à la séparation : Danièle}

Pour Danièle, le déclic s'est produit il y a bien longtemps, à la suite de la gifle ${ }^{15}$ reçue pour avoir mis à son seul nom la maison qu'elle avait ellemême payée, et à la suite de laquelle elle a porté plainte contre son compagnon, l'avertissant qu'il n'y aurait «pas de deuxième fois ». Cet événement a sans doute été si déterminant et reste si actuel pour elle, qu'elle n'a pas pu entendre la consigne de l'enquêtrice, lors de l'enquête téléphonique, et a situé ce fait dans «les douze derniers mois », alors qu'il s'est produit en $1986^{16}$. Il n'y a effectivement pas eu de «deuxième fois »,

\footnotetext{
${ }^{15}$ Danièle parle d'une gifle, mais, comme fréquemment au cours de l'entretien enregistré, elle a sans doute minimisé les faits : cela semble avoir été plus qu'une «simple » gifle, car elle ajoute : «J'ai été à la gendarmerie, j'ai vu que je n'aurais pas pu faire face... ».

${ }^{16}$ Voir, dans ce numéro : «De l'enquête statistique à l'enquête sur les sorties de la violence conjugale : constitution de l'échantillon et démarche d'enquête ».
} 
du moins pour elle, car elle n'a pas réussi à préserver ses enfants de la violence de leur père et beau-père. Elle n'a pas réussi non plus à ce que son compagnon ne parte pas, sans rien lui en dire, pour des périodes plus ou moins longues chez d'autres partenaires et ne fasse pas des enfants « dehors ».

La gifle a été le déclic qui lui a permis, en portant immédiatement plainte, d'éloigner d'elle depuis longtemps les violences physiques. Mais, pour mettre fin aux souffrances engendrées par les violences psychologiques et les atteintes de son conjoint à la relation conjugale, dont elle laisse entendre qu'elle souffre beaucoup plus qu'elle ne veut bien le dire et qui semblent s'être nettement aggravées, il lui faudra mettre fin à cette relation, ce à quoi elle se prépare, lentement et douloureusement, en cultivant l'indifférence à son égard.

Elle avait réussi jusqu'alors à se résigner à «faire avec ce qu'elle avait », puisque, dit-elle, «l'homme n'est pas parfait», mais les brutalités commises récemment à l'égard de leur fille malade semblent avoir constitué un second déclic, la prise de conscience de l'échec irrémédiable de la relation, si bien qu'ils évitent maintenant de se trouver non seulement dans la même pièce, mais également au même étage de la maison. Propriétaire de celle-ci, elle lui a d'ailleurs déjà dit de partir. Et, l'ayant « déjà quitté dans sa tête », en raison de ses infidélités et de la violence qu'il continue à manifester à l'égard des enfants, elle « apprend à devenir indifférente », ce qui lui permettra non seulement, comme aujourd'hui, de refuser, pour « garder sa dignité », de lui montrer qu'elle le désire, mais aussi de ne plus le désirer ${ }^{17}$.

\section{B. - Accepter le divorce et se sentir renaître}

Après le déclic qu'a provoqué chez Kelly la lecture du «Harcèlement moral ${ }^{18}$, elle «s'accorde » six mois pour s'occuper de sa mère malade, mais surtout, peut-être, d'elle-même :

Alors je vais chez elle, et je me suis accordé six mois pour m'occuper d'elle et moi-même 'sortie du verre' - parce que quand on est dans un verre et qu'on secoue le verre on est en pleine tempête, alors que bon, en sortant, on se rend compte qu'on

\footnotetext{
${ }^{17}$ Notons que, parmi les femmes rencontrées, Danièle est la seule qui ait fait explicitement mention d'un désir physique ressenti à l'égard de son compagnon ou partenaire.

${ }^{18}$ Marie-France Hirigoyen. Le harcèlement moral : la violence perverse au quotidien. Paris, la Découverte \& Syros, 1998.
} 
est dans un verre. Donc, pour avoir le recul, il fallait absolument que je coupe court. En allant chez ma mère, j'ai eu l'impression de sortir de ma cage et plus du tout l'envie de rentrer dans ma cage. Et c'est là que le pasteur a servi de médiateur et de négociateur. Il [son mari] a prétendu qu'il ne voyait pas ce qu'il faisait [de mal]. Moi, j'ai dit : 'Je vois et je ne retourne pas'.

Mais son mari la menace de demander le divorce, chose qu'elle n'avait pas envisagée et qu'elle rejette tout d'abord, parce qu'elle a trop souffert elle-même de la séparation de ses parents lorsqu'elle avait cinq ans - ce qui l'a fait brutalement passer alors « de l'enfance à l'âge adulte» :

Mon mari a dit: 'Si tu ne reviens pas, je fais une demande de divorce'. C'était là le chantage, je ne pensais pas qu'il irait jusqu'au bout et il a été jusqu'au bout de sa menace, ça m'a arraché le cœur. J'avais toujours dit: 'Jamais le divorce', parce que ma mère est divorcée, c'est pas une maladie héréditaire! (...) C'est-à-dire que je souhaitais me séparer de lui et que lui finisse par prendre conscience de son état, de sa situation, pour voir ce qu'il peut faire pour que j'aille mieux. Puisque, dans mon schéma mental, je n'avais jamais envisagé le divorce.

C'est cependant «le jour où elle a accepté le divorce » qu'elle s'est sentie libérée :

Le divorce, pour moi, a été prononcé le jour où j'ai accepté le divorce. Ça veut dire que la prononciation du divorce n'a fait que ratifier quelque chose, une fois qu'on a accepté. (...) Je me suis sentie légère. Et j'ai fait mon adolescence, et ma petite enfance, tout ce que je n'avais pas vécu. J'ai eu l'impression de renaître. C'est-à-dire, tout ce dont j'avais été privée avant, je l'ai vécu à ce moment-là...

\section{C. - Accepter le divorce et rester respectable : Emmanuelle}

Emmanuelle n'avait jamais eu de problèmes avec son mari «au niveau de l'amour qu'on avait l'un pour l'autre », lui reprochant seulement 
de ne pas la défendre face aux attaques incessantes de sa belle-mère. Mais, lorsque, il y a quatre ans et après sept ans de mariage, il rencontre une autre femme et veut «vivre autre chose », il «devient méchant, très méchant » pour l'obliger à accepter le divorce.

Elle ne s'y oppose pas, mais le prévient qu'elle-même ne fera aucune démarche en ce sens. Elle lui suggère cependant de donner comme motif l'incompatibilité d'humeur. Mais, bien qu'il affirme n'avoir « absolument rien à lui reprocher », il intente une procédure pour faute grave et tient à inscrire comme motif... qu'il n'accomplissait pas le devoir conjugal à cause de l'état de santé de sa femme. En raison vraisemblablement du principe selon lequel «nul ne peut se prévaloir de sa propre turpitude », et peut-être aussi de la «cruauté particulière » que représenterait le divorce étant donné la santé de sa femme, qui venait de subir plusieurs opérations graves en quelques mois, le divorce lui est refusé et le juge n'accepte qu'une séparation légale.

Comme Vanessa et Alexie, Emmanuelle n'avait pas eu d'autre partenaire que son conjoint. Mais, si les deux premières ne pouvaient se résoudre à « lâcher prise » et à accepter ou initier une rupture qui leur aurait coûté la «respectabilité » à laquelle elles aspiraient, c'est la «noblesse » avec laquelle elle a accepté un divorce que le juge a finalement refusé d'accorder à son mari qui a consacré la « respectabilité » d'Emmanuelle. Même s'ils ont quelquefois douté de la réalité ou de la gravité des violences qu'il lui avait fait subir, tous les amis de son mari ont été choqués qu'il laisse ainsi tomber «méchamment» une femme gravement malade, qui lui avait toujours été complètement dévouée, en faisant même «trop » à leur avis, qui n'avait aucune famille ni amis personnels en Martinique et qui venait de perdre le grand-père qui l'avait élevée en Guyane et auquel elle était très attachée. Comme le juge, ils ont tous «été contre lui » et, depuis, aident «moralement» Emmanuelle - mais, son mari versant rarement la pension alimentaire à laquelle il a été condamné, ils l'aident aussi matériellement par: « des coups de main, des fruits, des légumes, un sachet de pâtes par ci, du poisson, beaucoup comme ça ».

\section{V. - PRENDRE L'INITIATIVE DE LA RUPTURE}

Outre les femmes d'origine étrangère (Christelle, Henriette, Nathalie, Nicole) qui ont fui leur conjoint après des coups et des menaces plus graves encore que d'habitude ou qui ont servi de déclic, quatre femmes ont pris l'initiative de la rupture, en quittant leur conjoint (Jeanne, Manuela, Sophie) ou en réussissant à lui faire quitter le territoire (Françoise). 


\section{A. - « Expulser » le conjoint violent : Françoise}

Françoise, infirmière d'origine métropolitaine, n'a jamais porté plainte contre son ami, un réfugié politique sud-américain, alcoolique, très jaloux et très violent, qui l'a suivie à la Martinique lorsqu'elle s'y est installée. Plusieurs scènes de violences graves ont pourtant donné lieu à l'intervention des forces de l'ordre :

Aux Trois-Ilets, ils lui ont fait juste une petite leçon de morale, et puis c'est tout, et à moi avec, parce que, quand on ne veut pas porter plainte, ça veut dire [pour eux, qu'on accepte]... Bon, à Fort-deFrance, c'est quelqu'un qui les a appelés parce qu'il était en train de me taper sur la Savane19. (...) Et puis les gendarmes [en fait, les policiers] sont arrivés. Ils ont essayé de le calmer [en vain] et donc ils l'ont emmené. J'avais vraiment peur, et eux n'ont pas cherché à me poser de questions. Ils sont partis avec lui et ils m'ont laissée là, comme ça, sur la Savane... Rien du tout, juste quand j'ai dit: 'J'ai peur', là, ils l'ont emmené, et c'est vrai que je ne me sentais pas de remonter avec lui... Le lendemain matin, il était fou. Parce que j'étais pas allée le chercher, j'étais pas allée le voir, je ne m'étais occupée de rien, parce que je n'avais pas téléphoné, donc il avait passé toute la nuit en garde à vue. (...) Je pense qu'à la fin, j'ai eu peur [pour ma vie]. Au début, non, et puis après, après... J'allais travailler avec des lunettes noires, puis j'ai arrêté d'aller travailler. Je le cachais, je le cachais. Après, j'ai caché carrément que cette relation durait.

Elle qui ne veut pas porter plainte parce que ce n'est pas «dans sa mentalité », recourra cependant à la menace implicite des forces de l'ordre pour réussir à « expulser» son conjoint, deux ans après leur arrivée, après avoir sollicité de sa sœur le prix du billet de retour :

Et puis, un jour, j'ai dit: stop. Donc, j'ai appelé ma sour, afin qu'elle m'envoie de l'argent - parce que je ne leur avais pas dit qu'il venait avec moi en Martinique, sinon, ils ne m'auraient pas laissée, alors je lui avais caché. Donc, j'ai dit à ma sœur:

\footnotetext{
${ }^{19}$ Grand espace vert et place centrale aménagée en bord de mer.
} 
'Envoie-moi de l'argent en Martinique, que je prenne un aller simple pour repartir'. Donc, elle m'a envoyé l'argent et il est parti. Ca n'a pas été facile. Jusqu'à l'aéroport, il m'a appelée: 'Je reste - Non!'... Oui, ben, sinon, tant pis, j'aurais été voir les gendarmes... Le fait qu'il soit réfugié politique, je pense qu'il [a eu peur].

Aujourd'hui, Françoise estime qu'elle «peut le remercier, pas pour les violences, mais au moins pour mon caractère de battante ». D'ailleurs, après avoir "laissé passer trois ans », elle le revoit de temps à autre et se réjouit d'avoir «au moins gagné quelque part », puisqu'il se met maintenant plein de garde-fous pour ne pas céder à la violence avec sa nouvelle compagne. Cependant, elle a «toujours cette crainte» et, lorsqu'elle va le voir, lui dit : «Si tu bois, je pars».

\section{B. - Partir malgré le risque du « crime passionnel» : Sophie, Jeanne}

Quand nous avons rencontré Sophie, sa situation avait changé depuis l'enquête téléphonique. Elle avait quitté son compagnon depuis deux mois, lasse de ses infidélités, des relations «ambiguës » qu'il entretenait avec les mères de ses enfants et de ses mensonges. Elle qui avait choisi, peu avant de vivre avec lui, de pratiquer sérieusement son catholicisme et faisait partie d'un groupe de prières, vivait en effet sa situation conjugale comme une « débauche », qui la mettait en contradiction avec elle-même, ses valeurs, sa religion. Après avoir quitté son compagnon, elle va d'abord chez une amie, puis part en pèlerinage pendant deux semaines en métropole. A son retour, elle emménage dans un appartement, dont seule l'amie chez qui elle s'était réfugiée connaissait l'adresse.

Son compagnon ne veut pas croire qu'elle l'a vraiment quitté et ne l'accepte pas, pense qu'elle va «avaler la pilule et revenir», d'autant qu'il ne l'a pas encore «vue avec quelqu'un », ce qui, pense-t-elle, le ferait passer à «la phase $2 »$ du harcèlement qu'il lui fait subir. Il la bombarde de messages téléphoniques, lui laissant, par exemple,

Trois messages à deux minutes d'intervalle: le premier, il pleure en me disant qu'il faut que je revienne, le deuxième, il va me supplier en disant que je suis une salope et le troisième, il va s'excuser.

Il lui laisse des mots sur son pare-brise, la suit au travail (où des collègues l'escortent quand elle sort tard), et, surtout, réussit à trouver son 
adresse, l'épie pendant des heures du parking ou des bosquets de la résidence et l'appelle pour commenter ce qu'elle fait. Ses «accès de folie », qui font peser sur elle la menace du crime dit « passionnel », inquiètent énormément Sophie, qui a fait part à ses proches de ses dernières volontés.

Ces craintes évoquées par Sophie devant le harcèlement qu'elle subit de la part d'un conjoint avec lequel elle ne veut plus vivre mais qui n'accepte pas qu'elle le quitte font malheureusement écho aux «faits divers » tragiques qui font trop souvent la une de France-Antilles. Sophie se souvient d'ailleurs que :

Les faits divers... Quand on vivait ensemble et qu'il y avait ces faits divers-là, et que je dis: 'Comment [peut-on faire ça] ?' Il me dit: 'Je comprends ces hommes-là...'. (...) Quand je l'ai rencontré [après l'avoir quitté], il m'a dit: 'Le pire, c'est que je vois que tu as l'air bien, tu t'amuses, t'as même pas perdu un gramme, comment ça se fait? - Attends, je lui dis, c'est grave, tu as envie de me voir dépérir et tu dis que tu m'aimes?'. Il voudrait me voir couchée dans la rue, je ne sais pas... (...) Je savais tout ça, je savais. Il me disait: 'J'ai pas fait tout ça pour que tu partes'. Il me disait toujours des petites paroles qui me faisaient prendre conscience que ce serait difficile de le quitter (...). Je savais que ce serait difficile ${ }^{20}$.

La menace de «crime passionnel » a également plané sur Jeanne, «partie en bigoudis » pour «sauver sa peau » après avoir vu le désir de meurtre dans les yeux de son compagnon. Celui-ci a d'ailleurs tenté de lui faire quitter la route au cours d'une course-poursuite en voiture.

\section{Dieu : Manuela \\ C. - Compter sur ses propres forces et sur l'aide de}

Victime d'attouchements à huit ans de la part d'un oncle, Manuela a appris tôt à ne pas compter sur le secours d'autrui : on lui a alors, en effet, intimé de «se taire au lieu de dire n'importe quoi ». Elle se «referme sur elle », cesse de porter des robes ou des jupes, et de se montrer « féminine ». La confiance qu'elle pourrait avoir en l'aide d'autrui n'est pas non plus encouragée par le refus de son père de l'aider à passer le permis de conduire,

\footnotetext{
${ }^{20}$ Souligné par nous.
} 
car elle a de moins bons résultats scolaires que son frère, puis de l'aider à assumer sa première grossesse - non plus que par le mauvais accueil que lui réservera sa famille lorsqu'elle décidera « sur un coup de tête » de quitter le père de son enfant, en raison de violences liées à ses infidélités :

A force de dire: 'Non, non, non', ça ne donne pas du tout l'envie de demander quoi que ce soit... Et puis après, [mon père] m'a demandé de me débrouiller par mes propres moyens, et j'ai fait la même chose avec les autres...

Malgré les grandes difficultés financières auxquelles elle se heurte, elle met sa fierté à refuser longtemps l'aide du père de ses enfants (ils en ont eu un deuxième depuis son départ, mais il en a eu également avec une autre femme). Bien que la relation ait continué, surtout «pour les enfants », elle a refusé de retourner vivre avec lui, se sentant «plus en sécurité» et « maîtrisant mieux » les choses en étant «chez elle». Elle finit cependant par le menacer d'aller en justice pour qu'il reconnaisse les enfants et verse des pensions alimentaires, ce qui semble l'avoir conduit à s'investir davantage dans leur éducation et leur entretien.

C'est cependant essentiellement sur ses propres forces et son courage qu'elle continue à compter - ainsi que sur l'aide de Dieu : elle se demande comment elle tiendrait si «elle n'avait pas la foi ».

\section{VI. - SI DIEU VEUT ...}

\section{A. - « Dieu à mes côtés » pour supporter : Valérie}

Pour nombre des femmes martiniquaises rencontrées à la suite de l'enquête téléphonique ${ }^{21}$, la religion aide à supporter des situations difficilement supportables, mais dont elles n'envisagent réellement de pouvoir sortir que par une espèce de miracle qui leur rendrait l'amour de leur conjoint ou ami, et rendrait enfin fidèle cet «homme qui aime les femmes». Même Danièle, qui dit pourtant ne pas être pratiquante et n'avoir fait baptiser ses enfants que pour ne pas risquer de devoir les enterrer sans qu'on lui accorde un office religieux, répond quand on lui demande où elle puise sa force :

C'est-à-dire que, dans toutes mes souffrances, je vais vous dire, j'ai toujours eu Dieu à mes côtés...

\footnotetext{
${ }^{21}$ Les femmes rencontrées par l'intermédiaire des institutions d'aide n'ont pas évoqué l'aide de Dieu comme recours, soit pour leur permettre de supporter la violence, soit pour les aider à partir.
} 
Et c'est la prière, « intensive » pourrait-on dire, qui empêche Valérie de se suicider :

Un jour, il m'a sorti - c'est dur comme mots 'Couman ou pa tan man pa léw ankò, man pa léw man pa léw'22. Il criait, il criait, il criait. Dire ça a quelqu'un, c'est fort. Alors je l'ai à moitié avalé, ça fait très mal. (...) C'est un truc qui est très fort. Très très fort, et un rejet, avec beaucoup de mots trop forts pour ma petite tête qui était déjà affaiblie! Eh bien je suis rentrée [après avoir] emmené les enfants à l'école. Le chauffeur m'a regardée, il a dî se dire: 'Tiens, qu'est-ce qu'elle a la petite dame, elle va faire un cas avec son corps [se suicider] ?' J'ai dit: 'Non, tu rentres chez toi, va prendre une douche'. [Arrivée à la maison], j'ai mis mes louanges le plus fort possible et je me suis mise à pleurer, à crier au Seigneur. J'avais fermé toute la maison. J'avais besoin de ça, de rentrer en communion avec quelqu'un qui pouvait m'aider, à qui je pouvais me confier... Oui, je lui ai dit: 'Est-ce qu'il pense que je suis son enfant, pour me faire souffrir comme ça ?' (...) Que j'ai besoin de sa force, il faut qu'il me booste, il faut qu'il m'amène là où il veut que j'aille. J'ai parlé, j'ai parlé, j'ai parlé, j'ai parlé... Après je pouvais soulever une montagne... (Maintenant), je n'ai plus envie d'en finir, parce que, quand ces choses, les pensées lugubres me viennent, je dis: 'Non, Seigneur, je suis là, je peux puiser dans Ta force, j'ai besoin de Toi !' Et, là, tout de suite, j'attrape mon livre et puis, c'est parti, je cherche, je tombe sur un psaume, je tombe sur une parole biblique qui me réconforte.

\section{B. - Changer le rapport de forces en s'appuyant sur la famille et la prière : Delphine et la parole libérée}

Delphine - à laquelle, à l'issue de l'enquête téléphonique, nous avions attribué le score global de violences conjugales le plus élevé de

\footnotetext{
${ }^{22}$ «Mais tu ne comprends pas que je ne veux plus de toi. Je ne veux plus de toi! Je ne veux plus de toi !».
} 
l'échantillon - constitue le seul cas, parmi les femmes rencontrées, de solution de «sortie » recherchée, au moins en première instance, dans autre chose que la rupture, la fuite de la victime ou l'exclusion du conjoint. C'est aussi le seul cas où la famille du conjoint ait pris fait et cause pour la femme victime.

Après que son mari lui ait interdit d'aller au groupe de chant et pris les clefs de la voiture pour l'en empêcher, Delphine s'était précipitée chez ses beaux-parents (le couple vit sur le terrain familial) pour les prendre à témoin et leur demander de la conduire à cette réunion, ce à quoi son mari s'est opposé, en les menaçant. Le mari d'une belle-sœur de Delphine, marraine de l'une de ses filles, prend alors l'initiative d'une grande réunion de famille, où assistent les trois enfants, leurs marraines et les conjoints de celles-ci. Ses propres parents (l'oncle et la tante qui l'ont élevée) ne sont pas présents physiquement, mais se sont « mis en réunion de prière »:

J'étais partie en réunion de caté, et, à mon retour, ils étaient déjà tous là. J'ai tout raconté, lui il s'est tu. Il s'est tu. En fait, il disait toujours: 'Ce n'est pas vrai, ce n'est pas vrai', et la marraine de la cadette a dit: 'Ah, oui, quand tu venais chez nous, tu l'insultais, tu lui disais toujours de se taire et tout'. Et puis y'a les autres, même les messieurs en parlaient aussi, ils ont dit: 'Ah oui, mais c'est toujours toi la parole, elle n'a droit à rien, elle doit rien dire, c'est toi qui dois acheter, c'est toi qui dois faire ci, elle n'achète rien, elle ne dit rien'. (...) Ben, en fait, on lui a dit (que) ce qu'il fait c'est pas bien, et qu'il faudrait qu'il me remette tout (l'argent) qu'il a pris.

Le « déballage » ne suffit pas à changer les choses. Mais, si l'aînée des filles n'a rien manifesté pendant la réunion de famille, car elle savait déjà à quoi s'en tenir et s'entendait mal avec son père, la cadette réagit violemment, vomit et veut frapper son père. Très perturbée, elle se confie par la suite à l'assistante sociale scolaire, qui convoque le père :

C'est à partir de là que ça a cessé. Ca a cessé un peu. Il s'est calmé. Pourquoi ? Parce que c'était déjà arrivé au sein de l'établissement scolaire. (...) La tension s'est apaisée et il s'est peut-être rendu compte de ce qu'il faisait.

D'elle-même, Delphine n'aurait fait aucune démarche vers les institutions, pour des raisons matérielles - 
Je sais, y'a les assistantes sociales, y'a des... Mais, en fait, tout se trouve sur Fort-de-France et on peut pas toujours se déplacer... -

mais, surtout par peur des représailles :

Pour qu'il n'y ait pas, par rapport aux enfants, parce que, bon, je suis là pour les protéger. Par rapport aux enfants, je voudrais pas qu'il se passe des choses désagréables. [?] Ben, que, aller à l'association des femmes battues, et après qu'il apprenne que je suis allée, et qu'il s'énerve... Oui, parce que, quand on apprend... Pas mal de personnes sont déjà allées à l'association femmes battues, et après leurs maris découvrent qu'elles sont allées... On se dit: 'Mais, par qui ces gens-là ont découvert que cette personne était allée ?' Et, deux jours après, le mari tue cette personne... ${ }^{23}$

Mais, après la réunion de famille et la convocation du mari par l'assistante sociale, la peur a disparu :

Là, je vois vraiment le changement. Il ne me dit plus rien. Je lui dis: 'Maintenant, je vais'. Je dis pas : 'Est-ce que je peux?'. Je dis : 'Je vais'. Il me dit rien. Parce que tout le monde sait ce qui s'est passé. (...) Je me sens apaisée. Par rapport à... Par exemple, il ne peut pas m'empêcher de prier, je continue mes prières et je me sens apaisée. Oui, ça va mieux. Parce que moi, j'ai décidé: 'Bon, c'est comme ça - et rien d'autre'. (...) Et, là, je pense que, même s'il a envie de dire quelque chose, il ne le dit pas... Oui, parce que je lui ai dit: 'Si tu continues, moi, je...' - Parce que plusieurs personnes m'ont dit de faire un dossier. Je dis: 'Je ne fais pas de dossier, je ne fais pas de dossier, c'est pas lui le Tout-Puissant, j'y crois pas, je ne fais pas de dossier. Parce que, en fait, là où j'en suis sortie, [c'est] parce qu'un jour j'ai senti que je ne pouvais plus tenir, mais plus tenir! J'ai senti que j'enfonçais. Je ne pouvais plus tenir, mes membres, rien du tout ne me tenait, je pouvais plus. Je sentais que je ne pouvais plus. Alors, j'ai

${ }^{23}$ Allusion à une affaire qui a déclenché beaucoup de polémiques. 
dit: 'C'est pas lui, le Tout-Puissant'. Il faut vraiment y croire... [Vous pensez que Dieu vous a aidée à sortir de cette situation?] Ah, oui! Je pense qu'il a été apaisé (...) Il ne m'insulte plus, il ne m'injurie plus, ça s'est apaisé, parce que moi, je fais ce que j'ai à faire, et le reste, pff... Parce que la famille a prié. Et moi, je les remercie parce qu'ils ont prié. (...) Moi, en fait, je suis là [et pas morte, comme Sandra] parce que j'ai eu le soutien de mes parents et de mes beaux-parents... ${ }^{24}$

«Pour l'instant», donc, Delphine a renversé le rapport de force conjugal, avec l'aide de la famille, de Dieu... et de l'assistante sociale scolaire. Elle n'envisage pas de partir, parce que les enfants ont besoin de leur père. Elle n'aime plus son mari, mais a de l'affection pour lui. Pour l'avenir?

On verra après. Je pense que quand les enfants vont grandir, je ne sais pas si je vais rester seule avec lui!

\section{VII. - CONCLUSION : LA DIFFICILE SORTIE DE LA VIOLENCE CONJUGALE DANS UNE SOCIETE D'INTERCONNAISSANCE}

$\mathrm{Au}$ terme de cet inventaire de parcours de sortie de la situation de violence conjugale - où certaines, comme Vanessa ou Alexie, en sont à peine aux premiers pas tandis que d'autres, comme Françoise, ont « tourné la page »-, on ne peut qu'être frappé par la différence des itinéraires empruntés par les femmes «migrantes » et par les « Martiniquaises ». Il ne semble pas en effet qu'on puisse imputer au seul hasard le fait que nous ayons rencontré les premières par l'intermédiaire de l'Union des Femmes de la Martinique, ou du CHRS Rosannie Soleil créé à l'initiative de cette association $^{25}$, et les secondes à l'issue d'une enquête téléphonique auprès

\footnotetext{
${ }^{24}$ Souligné par nous.

${ }^{25}$ Rappelons que les femmes rencontrées par l'intermédiaire du CHRS ou de l'UFM sont toutes, à un degré divers, des «étrangères » ou des «migrantes »: Henriette, Nathalie, Thérèse, Christelle et Nicole sont originaires des états voisins de la Caraïbe. Anne est née dans la péninsule ibérique, a immigré en France enfant et ne vit en Martinique que depuis peu. Magali est née en métropole d'une mère martiniquaise, qu'elle n'a pas vraiment connue puisqu'elle est décédée quand Magali avait trois ans, et d'un père originaire du Moyen-Orient, et elle ne semble pas avoir vécu en Martinique avant de s'y réfugier. Jeanne, certes, est née et a grandi en Martinique, mais l'essentiel de sa vie conjugale s'est déroulé dans l'hexagone et elle ne voulait pas revenir vivre dans un pays où elle «ne reconnaissait plus rien ».
} 
d'un échantillon aléatoire ${ }^{26}$. Certes, de nombreuses Martiniquaises s'adressent à l'Union des Femmes pour obtenir aides et conseils et certaines sont ou ont été hébergées par le CHRS. Certes, des femmes originaires des états voisins de la Caraïbe ou de l'hexagone parviennent, comme Françoise, à se libérer de la relation violente sans s'adresser à ces institutions.

Mais on ne peut négliger le rôle de l'interconnaissance et des réseaux familiaux et sociaux dans la société martiniquaise. Facteurs qui pèsent différemment sur les migrantes et sur les autres. Isolées du fait de leur migration et de l'enfermement moral et/ou matériel dans lequel les tient leur conjoint, sans famille en Martinique (ou ne pouvant attendre réellement d'aide de proches dans une situation identique à la leur ${ }^{27}$ ), n'y «connaissant personne », ne pouvant compter sur leur famille restée au pays (à laquelle elles ont souvent laissé la charge de leurs premiers nés), n'ayant pas ou peu de ressources propres, maîtrisant souvent mal la langue, les premières ne peuvent guère trouver d'aide que dans les structures d'aide sociale - même si, comme le montrent les cas de Nathalie ou de Nicole, leurs interlocutrices s'y montrent souvent défaillantes - ou dans le réseau associatif ${ }^{28}$.

Si c'est la faiblesse de leur réseau social et familial en Martinique qui représente l'un des principaux obstacles auxquels se heurtent les migrantes pour sortir de situations qu'elles ne veulent plus accepter, le poids des réseaux d'interconnaissance empêche, au contraire, bien souvent, les natives de Martinique qui n'ont pas connu de parcours migratoire (ou un bref épisode) de chercher auprès des institutions étatiques ou associatives l'aide que leurs proches ne veulent pas non plus leur fournir ou qu'ils ne sont pas en mesure de leur apporter.

Parmi les non migrantes rencontrées, seules Valérie et Delphine ont, en effet, obtenu une aide de leur famille. Les frères et sœurs de Valérie sont

\footnotetext{
${ }^{26}$ Parmi elle, pas de femmes d'origine étrangère, une seule «métro », Françoise, et une femme originaire de Guyane, Emmanuelle. Manuela et Alexie ont toutefois passé une partie de leur enfance dans l'hexagone.

${ }^{27}$ C'est même Nathalie qui aide sa sœur, venue elle aussi à la Martinique où elle a « tourné dans l'alcool », à repartir à la Dominique avec ses enfants. La mère de Christelle, également victime de graves violences conjugales, n'avait pu que lancer en vain un avis de recherche lorsque sa fille, mineure, avait disparu ; lorsqu'elle la rencontre par hasard, elle ne peut que lui indiquer une connaissance qui lui offrira l'asile dans un lieu moins insalubre que celui où elle s'est réfugiée après avoir fui son conjoint, et l'aider par la suite dans la mesure de ses faibles moyens financiers.

${ }^{28}$ A l'exception de Nicole, une femme « de niveau universitaire » et qui faisait partie dans son pays d'une association de lutte contre les violences conjugales, les femmes d'origine étrangère ont été adressées à l'UFM - dont, elles ne connaissaient pas l'existence - ou au CHRS par des assistantes sociales. Jeanne, qui, elle aussi, a fait des études supérieures, s'est adressée directement à l'UFM.
} 
plusieurs fois «venus la chercher» quand la situation était «hyperviolente» et remplissent ses placards et son réfrigérateur quand ils apprennent qu'elle n'a plus rien à donner à manger à ses enfants. L'aide accordée, bien tardivement, par les parents adoptifs de Delphine, qui se sont «mis en prières », et par la famille de son conjoint, qui a pris l'initiative d'un «conseil de famille», l'a sans doute été d'autant moins difficilement qu'elle n'envisageait pas de quitter son mari ${ }^{29}$. Il n'est d'ailleurs pas impossible que cette initiative ait été prise en partie pour permettre au couple de «laver son linge sale en famille» et éviter que les révélations de Delphine débordent le cadre familial, ce qui arrivera néanmoins lorsque l'une des enfants se confiera à l'assistante sociale scolaire. Delphine remarque d'ailleurs que "c'est à partir de là que ça a cessé, que ça s'est calmé. Parce que c'était déjà arrivé au sein de l'établissement scolaire ». Ce n'est que lorsque la respectabilité de l'homme est mise à mal sur la place publique qu'il «se tient tranquille», parce que «tout le monde sait ce qu'il s'est passé ${ }^{30}$. La publicisation de la situation a changé le rapport de forces dans le couple et permet à Delphine d'espérer pouvoir rester avec son mari au moins jusqu'à ce que leurs enfants quittent le foyer.

Le plus souvent, les victimes cherchent, par honte, à cacher leur situation à leur famille (vivant sur le terrain de sa belle-famille, Delphine n'avait ainsi jamais rien dit de ce qu'elle endurait avant que le refus de son mari de la laisser aller au groupe de chant ne «déclenche tout») ou ne sont pas en mesure de la solliciter, comme les femmes d'origine étrangère, mais aussi comme les «Martiniquaises» qui ont vécu des situations familiales

\footnotetext{
${ }^{29}$ Mais les beaux-parents de Delphine n'assistent pas à la réunion familiale où elle peut « vider son sac ». Elle le leur reprochera : «C'est pas des autres [dont] j'avais besoin, mais de vous, pour savoir comment votre fils fonctionnait $»$.

${ }^{30}$ Dans l'analyse qu'elle propose de son expérience d'un an d'écoute et d'observation d'hommes auteurs de violence conjugale astreints, dans le cadre de l'obligation de soins, à participer à un groupe de parole, Nadège Séverac montre la perte de respectabilité et la disqualification sociale provoquées par la révélation et la condamnation des violences exercées par l'homme sur sa conjointe : «La punition s'assortit d'une assignation identitaire qui au statut d'homme respectable (et en droit de 'laver son linge sale en famille') substitue celui, insupportable, de 'criminel' (selon leur propre terme). Autant de chocs et de ruptures qui marquent le désaveu social de la violence et viennent signifier très clairement l'interdit d'y recourir ». Elle montre aussi que cet interdit n'est le plus souvent intériorisé que comme «une question pratique» et non comme une «question morale» par des hommes qui éprouvent de grandes difficultés à se représenter la souffrance de leur victime et la dimension (a)morale de leur comportement, dans lequel ils ne parviennent pas à voir autre chose qu'une «erreur » pour laquelle il leur faut payer, dans une société qui s'incline devant «le droit des femmes ». («Hommes auteurs de violence conjugale : le pari de l'émancipation », in Daniel Weltzer-Lang \& Chantal Zaouche Gaudron (dir.). Masculinités : état des lieux. Toulouse, Eres, 2011, p. 255-265).
} 
difficiles dans leur enfance ${ }^{31}$, ce qui n'a pas favorisé la construction de liens forts dans la famille. Par ailleurs, du fait de l'impératif social de « respectabilité » féminine, appuyé par le poids du facteur religieux en Martinique, le recours à «la grâce de Dieu » ne fonctionne pas toujours, comme pour Delphine, dans le sens de la libération de la parole et de la sortie de la violence.

En légitimant des représentations telles que «la femme doit être soumise à son mari » et «sa place est auprès de lui », l'influence du christianisme et le contrôle social qui s'exerce dans les très nombreuses communautés religieuses présentes en Martinique, expliquent sans doute pour une part la «discrétion » des proches, qui refusent de s'immiscer dans la vie privée du couple et dans les situations de violence conjugale. Certes, la communauté religieuse de Magali l'a aidée à fuir son mari, à récupérer ses enfants et à s'installer à la Martinique, mais la raison invoquée par elle pour mettre fin à la relation a dû apparaître tellement grave à cette communauté qu'il lui est sans doute apparu légitime de tout mettre en œuvre pour qu'elle et ses enfants soient à l'abri de ce «damné »...

Lorsqu'elles veulent quitter leur mari ou compagnon, les femmes se heurtent ainsi bien souvent à l'incompréhension, si ce n'est à l'opposition de leur famille, qui les regarde de ce fait comme fautives, voire « délinquantes ». A l'exception de Manuela, qui y a d'ailleurs été mal reçue et n'y est pas restée longtemps, nous n'avons pas rencontré de situation où la femme s'est réfugiée dans sa famille. Certes, Kelly a fui son mari après la lecture du « Harcèlement moral » en allant vivre six mois chez sa mère, mais c'était parce que celle-ci était gravement malade.

Jeanne voit sa famille, «déçue de son comportement», lui reprocher, lors de sa dépression, de se «complaire dans le malheur» alors qu'elle a «tout pour être heureuse », puis se retourner contre elle, estimant qu'elle «fait bien des histoires pour rien » et qu'elle aurait pu «supporter davantage », comme sa mère - laquelle s'effondre en larmes quand, un soir de 31 décembre, elle voit débarquer sa fille «le dos zébré et les cuisses griffées », mais ne renonce pas pour autant à sortir pour le réveillon (la sœur de Jeanne, elle, l'emmène au commissariat pour tenter, en vain, de porter plainte, mais lui fait ensuite «subir » dans son église le réveillon auquel elle non plus ne veut pas renoncer). Hébergée au CHRS après avoir quitté le

\footnotetext{
${ }^{31}$ Ce qui est le cas de presque toutes : Danièle, Delphine, Emmanuelle, Alexie et Vanessa ont été «confiées» pour des périodes plus ou moins longues, à d'autres membres de la famille, voire à des inconnus, Valérie a perdu sa mère à 13 ans, Sophie est une «enfant dehors », le père d'Alexie était très violent, comme les partenaires de la mère de Vanessa, comme le beaupère de Danièle, comme la mère de Vanessa et celle d'Emmanuelle. Seule Stéphanie parle d'une enfance heureuse, mais ses parents étant aujourd'hui décédés, elle ne peut faire appel à eux.
} 
domicile conjugal, Jeanne voit «tout le monde s'éloigner », parce que, ditelle :

On est dégoûté de moi - le mot n'est pas trop fort, c'est ce que je ressens... On ne me côtoie pas : je suis tombée dans la délinquance. (...) Je quitte un endroit protecteur où j'étais bien, où j'avais tout ce que je voulais, où j'étais socialement... Comment dire ça? J'avais une place de choix, hein, pour aller me mettre dans une galère...

Donc: 'faut pas compter sur [nous] pour t'en sortir, quoi! T'as quitté le paradis pour te mettre dans l'enfer, dans la merde, donc, débrouille-toi!'

Sophie, dont le compagnon ne comprenait pas qu'elle ne soit pas heureuse, «avec le matériel» qu'elle avait, se heurte aussi à l'incompréhension de la famille de ce compagnon lorsqu'elle décide de le quitter : ils n'arrivent à s'expliquer une telle décision qu'en lui imputant, à tort, un nouvel amant, car elle avait « tout le confort, une grande maison » et, pensent-ils, « avec tout ce confort-là, on ne peut pas partir » ${ }^{32}$.

Ce n'est pas seulement à travers le conformisme social qu'elle induit que l'interconnaissance qui caractérise leur société pèse sur les Martiniquaises, censées supporter l'insupportable plutôt que de perdre le confort matériel et la respectabilité que leur assurent leur relation conjugale. C'est aussi à travers la crainte que, dans une telle société, où, dit-on fréquemment, «tout le monde connaît tout le monde » ${ }^{33}$, leurs démarches ne puissent pas passer inaperçues et rester confidentielles.

Parmi les femmes rencontrées à la suite de l'enquête téléphonique, certaines ont dit ne pas connaître l'existence de l'UFM, malgré l'activité et la médiatisation de cette association. D'autres, ne se sentant pas, ou ne se voulant pas concernées, n'ont pas «fait attention» à ses messages, l'assimilant à une association destinée aux seules «femmes battues». Stéphanie, qui n'est pas «battue », a ainsi répondu qu'il ne lui était même

\footnotetext{
${ }^{32}$ Dans le cadre d'une autre recherche effectuée par le CRPLC, une jeune femme hébergée au CHRS avait raconté que les gendarmes intervenus pour mettre fin à une scène de violences graves et auxquels elle faisait part de sa volonté de quitter son conjoint avaient tenté de l'en dissuader en lui disant: «Réfléchissez bien, vous avez une belle maison! (Témoignage recueilli dans le cadre de l'enquête réalisée par Patrick Bruneteaux, Justin Daniel, Joëlle Kabile, Nadine Lefaucheur et Véronique Rochais, Pauvreté, précarité et formes d'exclusion en Martinique : une approche qualitative, rapport pour le ministère de l'outre- Mer, janvier 2007).

${ }^{33}$ Il serait plus juste de dire : « où tout le monde est susceptible de connaître quelqu'un qui vous connaît ».
} 
«jamais venu à l'esprit » de prendre contact avec des associations (ou avec des assistantes sociales).

Les autres n'envisageaient pas de s'y rendre, par crainte des représailles qui, selon elles, ne manqueraient pas de se produire dans une société d'interconnaissance où « on ne peut rien cacher »-ni, d'ailleurs, se cacher $^{34}$. Delphine évoque ainsi les «faits divers» tragiques («Sandra, l'autre femme au Lamentin, l'année dernière il y a eu plusieurs femmes de tuées, alors, bon, ben...») pour expliquer sa méfiance à l'égard des institutions («car, Sandra, elle aussi était partie vers [s'était réfugiée chez] sa mère, et malgré tout...»). Les femmes rencontrées semblent ainsi souvent compter davantage sur la prière et sur la «grâce de Dieu » que sur la police ou la gendarmerie ${ }^{35}$.

On peut d'ailleurs s'interroger, particulièrement dans le contexte insulaire d'interconnaissance qui est celui de la Martinique, sur l'impact de la médiatisation des fémicides conjugaux - ou des modalités de cette médiatisation - sur les femmes victimes ${ }^{36}$, mais aussi sur leurs conjoints, qui sont parfois fascinés par ces faits divers et, comme le compagnon de Sophie, «comprennent» leurs auteurs. Si une telle médiatisation a le mérite de braquer le projecteur sur la gravité des violences conjugales dans la société martiniquaise, on peut aussi se demander si elle ne fonctionne pas, au final, comme un outil de «terrorisme patriarcal» intimant aux femmes l'ordre de rester à la merci de leurs bourreaux si elles ne veulent pas «finir brûlée comme Sandra ».

Le message que les femmes victimes reçoivent d'une médiatisation qui n'aborde le thème des violences conjugales qu'à travers les faits divers tragiques qu'elles produisent - laissant dans l'ombre les cas, heureusement plus nombreux, où les interventions policières, judiciaires, sociales et associatives parviennent à mettre femmes et enfants à l'abri de ces violences, - n'est-il pas, en effet, que les institutions censées leur venir en aide sont

\footnotetext{
${ }^{34}$ Emmanuelle, qui ne voulait pas en entendre parler, s'est finalement laissée convaincre par une amie de contacter l'UFM - où elle s'est sentie «entendue et très à l'aise ». Mais elle ne courrait pas alors de réel danger : ce n'était pas elle qui voulait quitter son mari, mais celui-ci qui voulait « reprendre sa liberté ».

35 Les entretiens ont été réalisés avant la mise en place de cellules spécialisées ou de personnels spécialement affectés à l'accueil des femmes victimes par la police ou la gendarmerie.

${ }^{36}$ Et sur leurs enfants : Manuela rapporte les réactions de son fils, qui a été témoin des brutalités commises par son père, lorsque le journal télévisé fait état des «crimes passionnels » : « Hier soir, quand il a entendu le bonhomme qui a mis le feu... Alors, en me regardant, il s'est mis à pleurer. Alors je lui demande ce qui lui arrive. Il me dit: 'Ah, je ne veux pas entendre parler de ça, j’aime pas voir ça...'. Du coup, quand ça passe, je suis obligée d'éteindre la télé ».
} 
incapables de les protéger du coutelas, du bidon d'essence ou du fusil d'un partenaire qui n'accepte pas d'être quitté ?

Certes, la médiatisation des crimes conjugaux renforce la visibilité de la violence conjugale et alerte à juste titre l'opinion publique. Mais, en faisant apparaître dans l'espace public des faits de violence extrême, elle y matérialise également des démonstrations de force genrées. Les «faits divers » les plus marquants de la dernière décennie en Martinique ont en effet concerné des femmes que leur conjoint ou ex-conjoint a abattues de façon spectaculaire sur la place publique (lieu de travail, parking fréquenté, etc.) - la «mise en images » à la une de France-Antilles ou dans le journal télévisé apparaissant comme le couronnement de la dimension spectaculaire, recherchée consciemment ou non par leur auteur, de ces «exécutions» conjugales. Reconnaissant les lieux, connaissant toujours «quelqu'un qui connaît quelqu'un » qui était proche, à un titre ou à un autre, de l'auteur ou de la victime, chacun et chacune, en Martinique, peut ainsi se sentir concerné par de telles «exécutions", dans lesquelles on peut voir, l'expression paroxystique d'une virilité ostentatoire, déjà repérée dans la socialisation masculine antillaise ${ }^{37}$. Dans un contexte de relations tendues entre les genres, le caractère spectaculaire des agressions de femmes dans l'espace public est porteur, d'un discours performatif, menaçant et insidieux - car implicite -, qui sonne comme un avertissement aux femmes qui voudraient s'échapper de la violence conjugale et de l'ordre sexué $e^{38}$ ou de la domination masculine que cette violence cherche à maintenir. S'il est bien évident que les structures de prévention et de répression ont à tenir compte de ce paramètre dans la gestion de leur communication autour de ces problématiques, ce qui est ici au cœur du débat, dépasse largement la question de la communication et concerne les modalités mêmes de la socialisation de genre aux Antilles.

\footnotetext{
${ }^{37}$ Sur ce point, rappelons que notre équipe a, parallèlement à l'enquête statistique Enveff, mené une recherche exploratoire sur la socialisation masculine aux Antilles, poursuivie actuellement dans le cadre des travaux du Groupe de recherches «Genre et société aux Antilles » (CRPLC). Voir, notamment, les communications de Roger Cantacuzène : «Le déficit d'expression de l'intime dans la socialisation masculine aux Antilles ", de Mylenn Zobda-Zébina: «La construction des marges de la masculinité aux Antilles », et, enfin, autour de l'affaire Pascale-Karen, celle de Joëlle Kabile: «L'homme antillais est irresponsable : jeux et enjeux des représentations féminines dans la construction de l'identité masculine antillaise», présentées au colloque international «Perspectives futures, en intervention, politiques et recherche sur les hommes et les masculinités, » mars 2011, Université Laval, Québec.

${ }^{38}$ Marylène Lieber. Genre, violences et espaces publics. La vulnérabilité des femmes en question. Paris, les Presses de Sciences Po, 2008.
} 


\section{RÉSUMÉ}

Nadine Lefaucheur, Joëlle Kabile et Léoncine Ozier-Lafontaine. Les itinéraires de sortie de la violence conjugale

Le renoncement des femmes à leur idéal familial, conjugal ou amoureux, ou à la « respectabilité » sociale que leur confère malgré tout leur relation conjugale, apparaît comme un préalable indispensable à leur engagement dans un itinéraire de sortie de la violence conjugale. Mais, à l'origine de celui-ci, il y a généralement un déclic qui, dans une prise de conscience, souvent lente et progressive, de l'anormalité de la situation vécue, fonctionne comme une révélation brutale qu'elles ne pourront plus la supporter, car « trop, c'est trop !».

Les migrantes rencontrées par l'intermédiaire d'une association féministe ou d'un centre d'hébergement ont, pour la plupart, pu sortir de la situation de violence grâce à ces institutions et aux assistantes sociales qui les avaient orientées vers elles ; mais, elles ont souvent, surtout si elles étaient originaires des états voisins de la Caraïbe, vécu auparavant une longue « galère » : sans ressources, sans réseau social, elles ne savaient ni à qui s'adresser ni ou fuir, pour elles et leurs enfants, la violence de leur conjoint.

Par méconnaissance ou par crainte que, dans une société d'interconnaissance, leurs démarches ne puissent rester confidentielles, les « Martiniquaises » rencontrées à la suite de l'enquête téléphonique s'étaient au contraire rarement adressées aux associations ou aux institutions policières ou sociales censées leur venir en aide. C'est parfois la résignation à une séparation ou à un divorce, d'abord refusés pour des raisons matérielles et/ou idéologiques, qui leur a permis de « renaître ». Parfois, au contraire, en dépit de la perte du confort matériel et de la respectabilité conjugale, elles ont pris l'initiative de la rupture pour fuir le risque d'un « crime passionnel » ou malgré la crainte d'un tel risque, paradoxalement accrue par la dénonciation médiatique des fémicides conjugaux. Mais, il est frappant de constater que, plus que sur l'aide de leurs proches ou des institutions, la plupart de ces femmes ne pensaient pouvoir compter, pour sortir de la violence conjugale, que sur leurs propres forces et sur l'aide de Dieu.

\footnotetext{
ABSTRACT

Nadine Lefaucheur, Joëlle Kabile and Léoncine Ozier-Lafontaine. Routes out of domestic violence

It appears that women's renunciation of their ideas about an ideal family, an ideal couple, or an ideal relationship, as well as their renunciation of the social "respectability" tied to those relationships, are prerequisites for their
} 
ability to leave situations of domestic violence. But behind the decision to leave, there is usually a trigger, or a slow and gradual awareness of the abnormality of their situation, that works to reveal that they cannot continue to endure the violence, because "enough is enough!"

Migrants recruited through a feminist organization and a shelter, with the support of these institutions and the social workers that they employ, have mostly left these situations of violence, but they often lived for a long time without resources or social networks, especially if they were from neighboring Caribbean states, and knew neither where to go nor where to flee for themselves and their children as they sought escape from their violent spouse.

In a society where most people know each other, fear that their efforts would not remain confidential kept Martinican women (encountered following the telephone survey) from contacting aid associations or social/police institutions meant to be of help. Despite the loss of material stability and the respectability of marriage, some took the initiative to break away from their relationships in order to avoid a "crime of passion" and did so despite their fear of taking such a risky step, a fear paradoxically increased by media exposure of spousal homicide. Other women initially resisted for financial and/or ideological reasons to a separation or divorce, and it was the act of giving in that allowed them to be "reborn." But it is striking that more than seeking help from relatives or institutions, most of these women thought they could only count on ending the violence by mobilizing their own resources and their faith in the assistance of God. 